\title{
PROJETO E ANÁLISE EXPERIMENTAL DE UMA NOVA ESTRUTURA DE RETIFICADOR HÍBRIDO TRIFÁSICO UTILIZANDO TÉCNICA DE COMPENSAÇÃO SÉRIE DE TENSÃO NO BARRAMENTO CC
}

\author{
Danillo B. Rodrigues ${ }^{1}$, Paulo R. Silva ${ }^{2}$, Luiz C. Freitas ${ }^{2}$, Gustavo B. Lima², Ernane A. A. Coelho², \\ Luiz C. G. Freitas ${ }^{2}$ \\ ${ }^{1}$ Universidade Federal do Triângulo Mineiro (UFTM), Departamento de Engenharia Elétrica (DEE), Uberaba - MG \\ ${ }^{2}$ Universidade Federal de Uberlândia (UFU), Núcleo de Pesquisa em Eletrônica de Potência (NUPEP), Uberlândia - MG \\ e-mails: danillorodrigues@yahoo.com.br,lcgfreitas@yahoo.com.br
}

\begin{abstract}
Resumo - Este trabalho apresenta a análise e o desenvolvimento de uma estrutura topológica de retificador híbrido trifásico que oferece as características operacionais de imposição de correntes de linha de entrada senoidais com elevado fator de potência e reduzida distorção harmônica de corrente, e de fornecimento de um barramento CC com tensão regulada utilizando a inovadora técnica de Compensação de Tensão Série no Barramento CC. Tais objetivos são alcançados tanto para condições normais de suprimento da rede elétrica como para condições de afundamentos temporários de tensão equilibrados e desequilibrados na rede CA. Para corroborar com a teoria exposta e com as análises de simulação computacional, um protótipo de $5 \mathrm{~kW}$ foi construído e avaliado em laboratório. Os resultados obtidos comprovam a eficácia da solução proposta e demonstram grande potencial para a alimentação de cargas CC sensíveis aos afundamentos de tensão na rede CA e para estabilização da tensão em microrredes CC devido à inerente intermitência das fontes de energia solar fotovoltaica e eólica.
\end{abstract}

Palavras-chave - Compensação Série de Tensão no Barramento CC, Correção de Fator de Potência, Distorção Harmônica, Microrredes.

\section{DESIGN AND EXPERIMENTAL ANALYSIS OF A NEW THREE-PHASE HYBRID RECTIFIER STRUCTURE USING A SERIES DC BUS VOLTAGE COMPENSATION TECHNIQUE}

\begin{abstract}
This work presents the analysis and the development of the topological structure of a Three Phase Hybrid Rectifier, which offers the operational characteristics for sinusoidal line current imposition with high power factor and low total harmonic distortion of the current. This structure also provides a de bus bar with regulated voltage using the innovative Series Voltage Compensation technique onto de bus for both normal condition of grid supplying as for conditions of temporary balanced and unbalanced voltage sags onto AC grid. In order to corroborate with the exposed theory and
\end{abstract}

computational simulation analyzes, a prototype of $5 \mathrm{~kW}$ was constructed and evaluated at laboratory. The results proved the efficacy of the proposed solution and demonstrated that it has great potential for application as power supply for DC loads suffering from voltage sags on the AC grid and for voltage stabilization on DC microgrids due to inherent intermittency of solar photovoltaic and wind energies.

Keywords - Harmonic Distortion, Microgrids, Power Factor Correction, Series Voltage Compensation on the DC Bus.

\section{INTRODUÇÃO}

A implementação de sistemas de distribuição em Corrente Continua $(\mathrm{CC})$ ligados à rede de Corrente Alternada $(\mathrm{CA})$ tem se demonstrado como uma ótima opção para modernos sistemas de distribuição de energia elétrica em níveis de baixa e média tensão, devido à facilidade de integração com diferentes fontes renováveis e sua maior eficiência [1] - [10]. Estes sistemas de distribuição requerem controle de tensão no barramento $\mathrm{CC}$ para equilibrar o fluxo de energia entre as fontes renováveis, cargas $\mathrm{CC}$ e a rede $\mathrm{CA}$.

Uma vez que as microrredes são compostas, em sua maioria, por sistemas fotovoltaicos e microgeradores eólicos, os quais dependem de condições ambientais como o nível de radiação solar e o vento, respectivamente, a geração de energia elétrica por parte dessas fontes renováveis de energia acaba sendo intermitente, acarretando em oscilações de tensão no barramento CC [10].

A redução de produção de energia elétrica e a consequente queda na tensão no barramento CC podem acarretar na atuação dos dispositivos de proteção de cargas CC especiais, como dispositivos de acionamento de velocidade variável, conectadas ao barramento em corrente contínua, implicando em desligamentos inoportunos e perdas de produção industrial. O excedente de energia, por sua vez, é responsável pela elevação da tensão no barramento $\mathrm{CC}$, danificando os equipamentos conectados à microrrede. Diante do exposto, um sistema de distribuição CC capaz de lidar com severos degraus de carga e a instabilidade natural das fontes renováveis deve ser implementado. Artigo submetido em 23/11/2017. Primeira revisão em 22/02/2018. Aceito
para publicação em 17/04/2018 por recomendação do Editor Marcello
Mezaroba. 
Diante dessa perspectiva, o presente trabalho se concentra no estudo de uma solução viável e eficiente para o controle da tensão no barramento de uma microrrede $\mathrm{CC}$, como apresentada, por exemplo, na Figura 1. Caso as fontes de energia renováveis não sejam suficientes para suprir as cargas $\mathrm{CC}$ ligadas ao barramento, essa falta de energia é compensada pela rede CA através do conversor CA-CC híbrido proposto. Nestas condições, a tensão no barramento CC é mantida a partir da potência fornecida pela rede CA mesmo diante de afundamentos temporários de tensão [11] - [15]. É importante salientar que, caso a energia fornecida pelas fontes renováveis seja maior do que as exigências das cargas, o excesso de energia gerado deve ser injetado na rede de distribuição em corrente alternada através de um inversor bidirecional, conforme já apresentado pelos autores em [16].

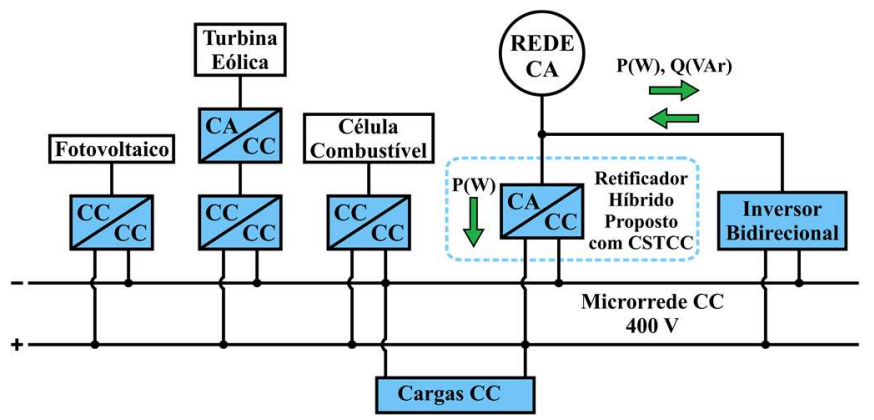

Fig. 1. Exemplo de um sistema de distribuição $\mathrm{CC}$ conectado à rede $\mathrm{CA}$.

A característica principal da solução proposta é a utilização de um único estágio de conversão CA-CC para proporcionar um barramento CC estável para conexão de cargas eletrônicas sensíveis a oscilações de tensão. Como nova contribuição, os autores apresentam uma estrutura Retificadora Híbrida Trifásica (RHT) que utiliza a técnica de Compensação Série de Tensão no Barramento CC (CSTCC). O diagrama esquemático da conexão do RHT proposto em uma microrrede CC é apresentado na Figura 2.

O arranjo do RHT proposto é constituído da combinação de dois grupos retificadores, conforme pode ser observado pela Figura 2. O primeiro é um retificador não controlado de seis pulsos com indutores do lado CA (Ret-1), e o segundo (Ret-2) é composto por um conversor Boost trifásico totalmente controlado com indutores do lado CA, ligado em paralelo com a rede elétrica e cascateado a um conversor CC-CC Full-Bridge isolado cuja saída é ligada em série com a saída de Ret-1, promovendo, desta forma, a compensação série de tensão no barramento $\mathrm{CC}$.

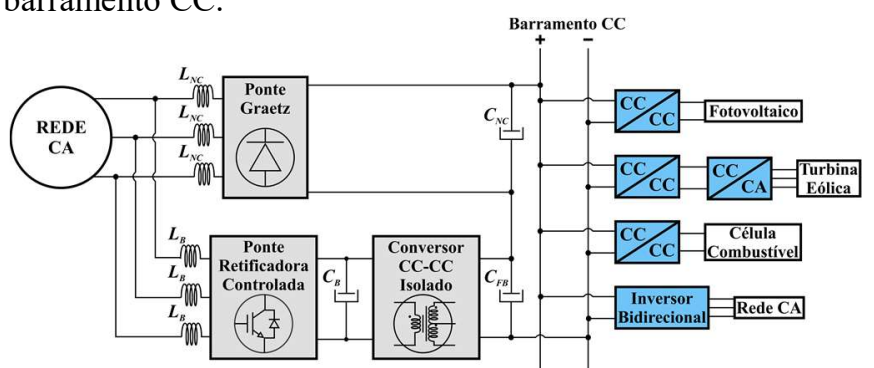

Fig. 2. Diagrama esquemático de uma microrrede $\mathrm{CC}$ com o retificador proposto usando um conversor Boost trifásico na conexão paralela de entrada e um conversor isolado CC-CC na conexão série de saída.
O RHT oferece duas características operacionais importantes: (1) a imposição de correntes de linha de entrada senoidais de forma a assegurar alto fator de potência e reduzida distorção hormônica total de corrente $\left(\mathrm{DHT}_{\mathrm{I}}\right)$; e (2) o fornecimento de um barramento $\mathrm{CC}$ constante de $400 \mathrm{~V}$, tanto para condições normais de suprimento da rede elétrica como para condições de afundamentos temporários de tensão equilibrados e desequilibrados na rede CA - conforme será demonstrado neste trabalho. Como principal diferencial em relação aos retificadores PWM convencionais, como por exemplo o conversor CA-CC Boost Bidirecional, destaca-se o processamento de potência do Ret-2 (conversor chaveado) o qual atinge no máximo $27 \%$ da potência nominal, promovendo redução de custos e robustez.

Neste cenário, a premissa principal de projeto é manter a divisão da potência entre os grupos retificadores de modo que, em condições normais de suprimento da rede elétrica, cerca de $73 \%$ da potência total de saída é processada por Ret-1, e $27 \%$ é processada por Ret-2. Este recurso oferece alta confiabilidade operacional e robustez, o que torna esta estrutura topológica muito atraente para aplicações em níveis de alta potência. Em outras palavras, isto significa que o retificador híbrido trifásico é uma estrutura que combina a robustez, simplicidade e confiabilidade do retificador trifásico de seis pulsos com a alta densidade de potência promovida pelos conversores chaveados.

\section{PRINCÍPIO DE OPERAÇÃO}

\section{A. Imposição de Correntes de Linha de Entrada}

Devido à sua característica de fonte de corrente na entrada, o conversor Boost trifásico totalmente controlado com indutores do lado CA é utilizado para impor correntes de linha de entrada senoidais, assegurando alto fator de potência e reduzida $\mathrm{DHT}_{\mathrm{I}}$.

As correntes de linha drenadas da rede CA pelo RHT são compostas pela soma entre as correntes requeridas por Ret-1 e as correntes impostas pelo conversor Boost que compõe a estrutura Ret-2, seguindo o mesmo princípio de imposição de corrente visto em [17]-[21]. Assim, as formas de onda das correntes de linha de entrada dependem das correntes impostas pelo conversor chaveado, as quais determinam a melhoria da DHT e a conformidade com as normas internacionais IEC 61000-3-2 e IEC 61000-3-4, conforme também pode ser observado em [22], [23].

\section{B. Compensação Série de Tensão no Barramento CC}

A compensação série de tensão se dá através da inserção do conversor Full-Bridge isolado com capacitor de saída $C_{F B}$ ligado em série com o capacitor de saída $C_{N C}$ do grupo retificador Ret-1, de modo que a soma das tensões em ambos os capacitores $\left(V_{C n c}\right.$ somado a $\left.V_{C f b}\right)$ constitui a tensão no barramento $\mathrm{CC}$, que por sua vez, é regulada em $400 \mathrm{~V}$. O conversor Boost, por sua vez, é controlado para fornecer uma tensão média fixa de $350 \mathrm{~V}$ na entrada do conversor FullBridge.

A Figura 3 apresenta o princípio de operação da compensação série de tensão para a condição normal de suprimento da rede CA de alimentação e para a condição de afundamento temporário de tensão. Para a condição normal de 
suprimento da rede elétrica, compreendida pelo intervalo de 0 até $t_{s a g}$, o retificador não controlado (RNC) fornece uma tensão média fixa através do capacitor $C_{N C}$ em torno de $293 \mathrm{~V}$ (tensão definida pelo valor médio das tensões de linha de entrada retificadas em seis pulsos), restando ao conversor Full-Bridge fornecer uma tensão média através do capacitor $C_{F B}$ de aproximadamente $107 \mathrm{~V}$, garantindo desta forma, uma tensão média fixa de $400 \mathrm{~V}$ no barramento $\mathrm{CC}$.

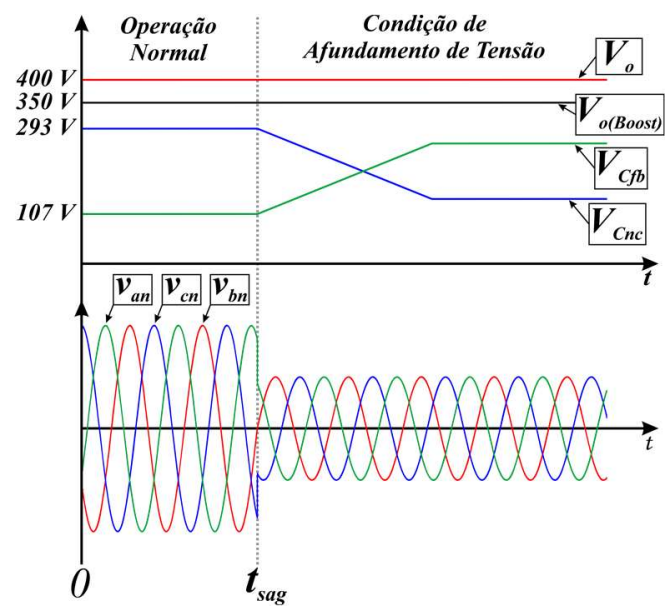

Fig. 3. Princípio de funcionamento da compensação série de tensão do barramento $\mathrm{CC}$.

Durante uma condição de afundamento temporário de tensão, compreendida pelo intervalo a partir de $t_{\text {sag }}$, devido à redução da tensão $V_{C n c}$ em função da queda da amplitude das tensões de alimentação da rede $\mathrm{CA}$, a tensão de saída do conversor Full-Bridge é elevada afim de compensar a queda de tensão no capacitor $C_{N C}$, garantindo desta forma, que a tensão no barramento CC seja regulada em $400 \mathrm{~V}$.

$\mathrm{Na}$ Figura 4 é apresentado um gráfico que demonstra o efeito do aumento da imposição da tensão no barramento CC sobre a divisão de processamento de potência entre as estruturas retificadoras Ret-1 e Ret-2. Nota-se que à medida que a tensão no barramento CC é aumentada de $300 \mathrm{~V}$ até 600 $\mathrm{V}$, ocorre um aumento no processamento de potência por parte da estrutura Ret-2, sendo que para uma tensão de $600 \mathrm{~V}$, a divisão de processamento é de aproximadamente $50 \%$ para cada estrutura retificadora. Isso demonstra que o processamento de potência por parte dos conversores chaveados é pequeno em termos da ampla faixa de tensão que o retificador híbrido trifásico pode controlar no barramento CC.

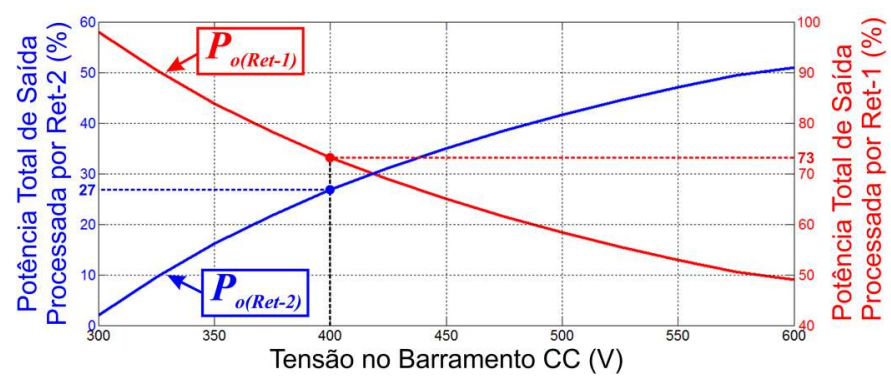

Fig. 4. Divisão de processamento de potência entre Ret-1 e Ret-2 com o aumento da tensão no barramento CC.
Para a aplicação mostrada neste trabalho, considerou-se como ponto de operação a imposição de uma tensão de $400 \mathrm{~V}$ no barramento CC o que implica na divisão do processamento da potência total entregue à carga em cerca de $73 \%$ para Ret-1 e $27 \%$ para Ret- 2 .

\section{ESTRATÉGIAS DE CONTROLE}

\section{A. Estratégia de Controle do Conversor Boost Trifásico}

O controle do conversor Boost baseia-se no controle da tensão no seu capacitor de saída a partir da imposição de correntes de linha de entrada senoidais. Para o correto funcionamento desta estratégia de controle, são realizadas sete aquisições de sinais: os sinais das correntes de linha de entrada $\left(i_{a(i n)}, i_{b(i n)}\right.$ e $\left.i_{c(i n)}\right)$; os sinais das tensões fase-neutro $\left(v_{a n}, v_{b n} \mathrm{e}\right.$ $\left.v_{c n}\right)$; e o sinal da tensão no capacitor $C_{B}\left(V_{o(B o o s t)}\right)$. É importante ressaltar que as aquisições das tensões de alimentação são realizadas para fins de referências de fase utilizadas pelos PLLs (Phase Lock Loop) visando estabelecer referências de corrente em fase com as tensões fase-neutro, conforme evidenciado na Figura 5.

Para o controle de tensão do conversor Boost, utiliza-se um controlador de tensão que processa o sinal de erro $e_{v(B)}$ entre a tensão de saída do conversor Boost, $V_{o(\text { Boost })}$, e a referência de tensão, $V_{o(B o o s t)}{ }^{*}$, definida em $350 \mathrm{~V}$. O sinal de saída $k_{v(B)}$ deste controlador de tensão é então multiplicado às referências senoidais digitais $i_{s e n-A} *, i_{s e n-B} *$ e $i_{s e n-C} *$ geradas pelos PLLs de cada fase, de forma a variar a amplitude das referências de corrente objetivando compensar qualquer variação na tensão $V_{o(B o o s t)}$. As referências finais de corrente $i_{\text {Ref-A }}, i_{R e f-B}$ e $i_{\text {Ref-C }}$ são determinadas, portanto, pela multiplicação de $i_{\text {sen-A }}{ }^{*}, i_{\text {sen-B } B} * \mathrm{e}$ $i_{\text {sen-C }}{ }^{*}$, respectivamente, pela saída $k_{v(B)}$ do controlador de tensão.

O controle de corrente, por sua vez, baseia-se na comparação entre as correntes de linha de entrada $i_{a(i n)}, i_{b(\text { in })} \mathrm{e}$ $i_{c(\text { in })}$ do RHT, obtidas a partir de sensores de corrente, e as referências finais de corrente $i_{\text {Ref-A }}, i_{R e f-B} \mathrm{e} i_{R e f-C}$, respectivamente. Essa comparação dá origem aos sinais de gatilho $V_{G S 4 B}, V_{G S 5 B}$ e $V_{G S 6 B}$ que são enviados às chaves $\mathrm{S}_{4 \mathrm{~B}}, \mathrm{~S}_{5 \mathrm{~B}}$ e $\mathrm{S}_{6 \mathrm{~B}}$, respectivamente. Como o funcionamento dos interruptores do conversor Boost ocorre de forma complementar, os sinais de gatilho de $S_{1 B}, S_{2 B}$ e $S_{3 B}\left(V_{G S I B}\right.$, $V_{G S 2 B}$ e $V_{G S 3 B}$ ) são gerados aplicando-se uma lógica not nos sinais de gatilho $V_{G S 4 B}, V_{G S 5 B}$ e $V_{G S 6 B}$, respectivamente.

Vale ressaltar que a implementação do controlador de corrente por Histerese se deu pela sua robustez e excelente resposta transitória [18]. Cabe destacar ainda que, com a frequência de amostragem ajustada em $50 \mathrm{kHz}$, a máxima frequência de comutação dos semicondutores do Conversor Boost é limitada em $25 \mathrm{kHz}$, evitando, portanto, frequências de comutação elevadas que poderiam levar à destruição dos semicondutores utilizados no projeto. 


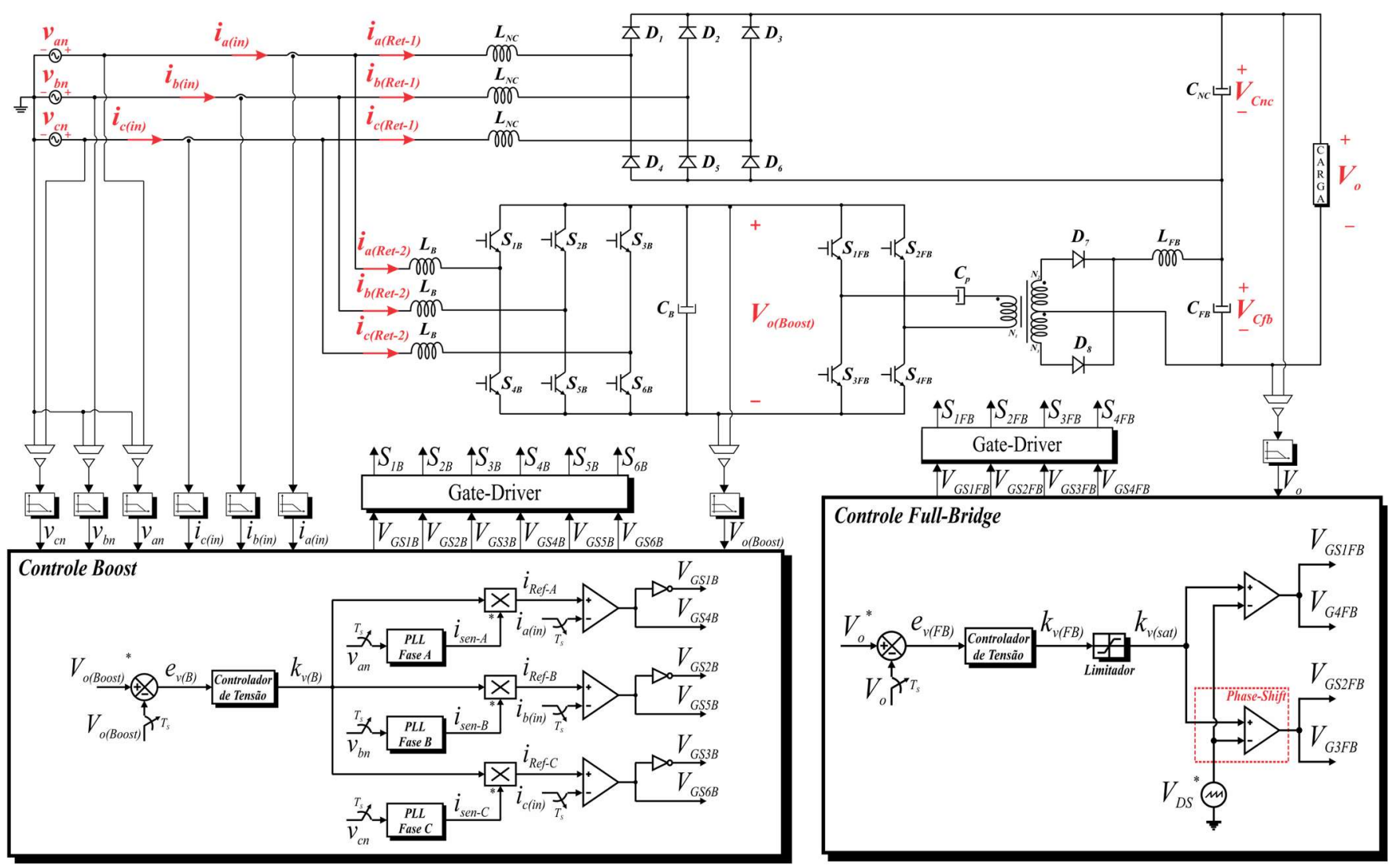

Fig. 5. Diagrama de controle do conversor Boost e do conversor Full-Bridge isolado.

\section{B. Estratégia de Controle do Conversor Full-Bridge}

Para promover uma tensão de $400 \mathrm{~V}$ no barramento CC, utiliza-se um controlador de tensão que processa o sinal de erro $e_{\nu(F B)}$ entre a tensão no barramento CC, $V_{o}$, e a referência de tensão, $V_{o}{ }^{*}$, definida em $400 \mathrm{~V}$. O controlador fornece um sinal de saída $k_{v(F B)}$ que é utilizado para a geração dos pulsos enviados aos interruptores $S_{I F B}, S_{2 F B}, S_{3 F B}$ e $S_{4 F B}$ do conversor Full-Bridge. O objetivo da lógica para geração dos pulsos dos interruptores do conversor Full-Bridge é reduzir o erro $e_{v(F B)} \mathrm{e}$ consequentemente estabelecer que a tensão no barramento CC siga a referência de tensão desejada.

A fim de evitar qualquer sobreposição entre os chaveamentos dos interruptores e garantir que a razão cíclica máxima de operação dos interruptores esteja abaixo de 0,5 para a metade do período de chaveamento $\left(T_{c h}\right)$, aplica-se um limitador sobre o sinal de saída $k_{v(F B)}$ do controlador de tensão. Para a geração dos sinais de pulso enviados às chaves $S_{I F B}$ $\left(V_{G S I F B}\right)$ e $S_{4 F B}\left(V_{G S 4 F B}\right)$, estabelece-se uma estratégia de controle baseada em modulação por largura de pulso (Comparação PWM - Pulse-Width Modulation), em que é realizada a comparação entre a saída $k_{v(F B)}$ saturada $\left(k_{\nu(s a t)}\right)$ e uma onda dente-de-serra de amplitude unitária e frequência de $25 \mathrm{kHz}\left(V_{D S}{ }^{*}\right)$.

Como a lógica de chaveamento de $S_{I F B}$ e $S_{4 F B}$ descrita anteriormente garante o trabalho destes interruptores para a primeira metade de $T_{c h}$, torna-se necessário estabelecer o chaveamento dos interruptores $S_{2 F B}$ e $S_{3 F B}$ para a segunda metade de $T_{c h}$. Para a geração dos pulsos enviados às chaves $S_{2 F B}\left(V_{G S 2 F B}\right)$ e $S_{3 F B}\left(V_{G S 3 F B}\right)$, primeiramente o sinal $k_{v(s a t)}$ passa por um módulo de comparação $P W M$ com deslocamento de fase (Comparação PWM com Phase Shift). O módulo de comparação PWM com Phase Shift acresce de 0,5 o sinal $k_{v(s a t)}$ e compara-o com a metade superior de $V_{D S}{ }^{*}$, garantindo que sinais de pulsos defasados em metade do período de chaveamento em relação os sinais $V_{G S I F B}$ e $V_{G S 4 F B}$ sejam enviados para os interruptores $S_{2 F B}$ e $S_{3 F B}$.

Destaca-se que o arranjo proposto garante um desacoplamento entre as estratégias de controle do conversor Boost e do conversor Full-Brigde. A malha de controle de tensão do conversor Boost realiza a regulação da tensão de entrada do conversor Full-Bridge em $350 \mathrm{~V}$. A malha de controle de tensão do conversor Full-Bridge, por sua vez, é responsável pela contribuição de tensão ao barramento $\mathrm{CC}$ na compensação série de tensão. Mesmo que as chaves do conversor Boost sejam desabilitadas (seja por uma falha no controle de imposição de corrente e regulação de tensão, por exemplo), o mesmo ainda continua operando como um retificador não controlado de seis pulsos, e o conversor FullBridge permanece alimentado (mesmo que com tensão inferior a $350 \mathrm{~V}$ ) e capaz de promover a compensação série de tensão em condições normais de operação.

\section{MODELAGEM MATEMÁTICA E PROJETO DOS CONTROLADORES}

Para o projeto dos controladores de tensão, primeiramente estabeleceu-se a modelagem matemática para a obtenção das representações matemáticas no espaço de estados referentes às etapas de operação dos conversores Full-Bridge e Boost.

Em seguida, utilizou-se as representações no espaço de estados para implementação de um método de integração numérico para solução dos vetores de estado e desta forma 
obter as formas de onda das variáveis de estados dos conversores. Logo, promoveu-se a utilização do método de integração trapezoidal para a solução do modelo matemático dos conversores Full-Bridge e Boost operando em malha aberta, e estabeleceu-se degraus nas variáveis manipuladas específicas de cada conversor a fim de se verificar o comportamento das variáveis controladas dos sistemas.

Com base nas respostas ao degrau obtidas, foi possível estimar as funções de transferências que definem o comportamento em malha aberta de cada conversor e, por consequência, os compensadores de tensão necessários.

\section{A. Projeto do Controlador de Tensão do Conversor Boost}

O diagrama de blocos da malha de controle do sistema equivalente do conversor Boost pode ser representado através da Figura 6, em que pode-se projetar o controlador de tensão com base na análise da função de transferência equivalente do sistema em malha aberta $G_{v i(e q)}(s)$ que relaciona a tensão controlada no capacitor de saída do conversor Boost $\left(V_{o(B o o s t)}(s)\right.$ - variável controlada) com a manipulação das amplitudes das correntes de linha $i_{a(\text { Ret-2) }}, i_{b(\text { Ret-2) }}$ e $i_{c(\text { Ret-2) }}$ impostas na entrada do conversor Boost a partir da variação das amplitudes das referências senoidais de corrente $i_{R e f-A}, i_{R e f-B}$ e $i_{R e f-C}\left(I_{R e f}(s)-\right.$ variável manipulada).
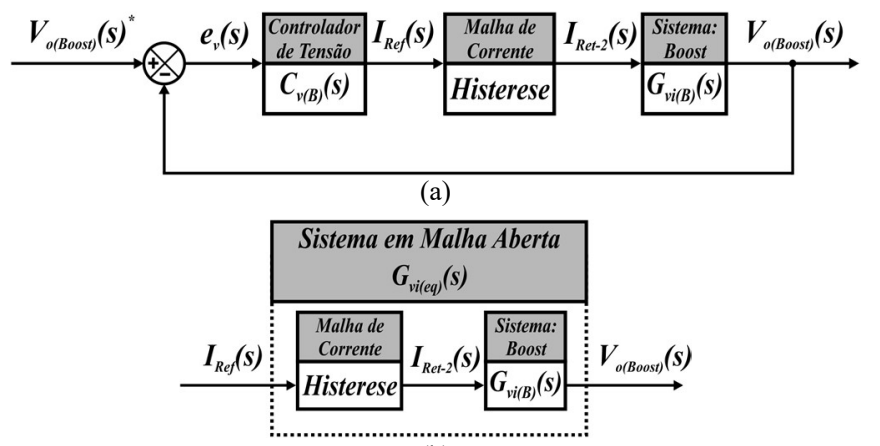

(b)

Fig. 6. Diagrama de blocos do sistema equivalente do conversor Boost: (a) em malha fechada e (b) em malha aberta.

Para a solução do modelo matemático e o consequente projeto do controlador de tensão, considerou-se o circuito equivalente apresentado na Figura 7 como sistema de análise. Para facilitar a análise, adotou-se a operação isolada do conversor Boost para a potência nominal da estrutura Ret-2 (27\% de $\left.P_{o}\right)$, trabalhando para impor correntes senoidais e regular a tensão de saída aplicada a uma carga de resistência $R_{F B}$, que por sua vez representa a resistência de carga equivalente à operação do conversor Full-Bridge. A resistência $R_{L b}$ representa a resistência dos enrolamentos dos indutores.

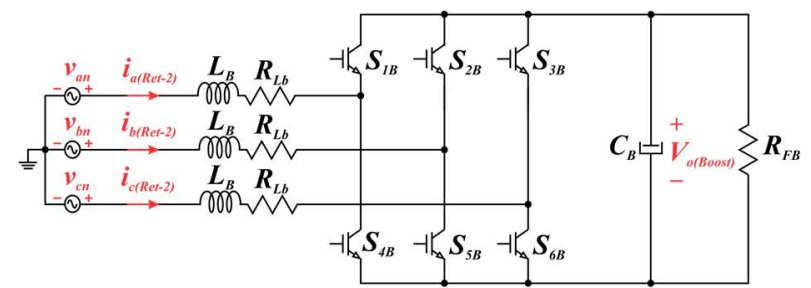

Fig. 7. Arranjo topológico equivalente do conversor Boost.
O objetivo foi, portanto, promover a solução do modelo matemático a partir da representação no espaço de estados do circuito da Figura 7 de tal maneira que permitisse estabelecer um degrau nas amplitudes das referências das correntes impostas e verificar o comportamento da tensão no capacitor de saída do conversor Boost.

Definidas as variáveis manipulada e controlada, implementou-se um algoritmo responsável por realizar o método de integração trapezoidal e desenvolver a solução do modelo matemático do conversor Boost operando em malha aberta.

Segundo a solução do modelo matemático, para a condição normal de suprimento da rede CA trifásica de alimentação, onde a estrutura Ret- 2 processa cerca de $27 \%$ da potência ativa total de saída, um valor igual a 5,05 para as amplitudes das correntes senoidais impostas nos indutores do conversor Boost $(\triangle A m p)$ permite a regulação da tensão no capacitor de saída em $350 \mathrm{~V}$.

Analisando a resposta da solução do modelo matemático apresentada na Figura 8, percebe-se que a resposta do sistema em malha aberta pode ser aproximada por uma resposta de sistema de controle de primeira ordem, onde as informações necessárias para a estimativa da função de transferência do sistema em malha aberta do conversor Boost, $G_{v i(e q)}(s)$, como o valor em regime permanente da tensão $V_{o(B o o s t)}$ após o degrau $\left(\Delta V_{o(B o o s t)(r p)}\right)$ e o tempo necessário para a resposta da tensão $V_{\text {o(Boost })}$ atingir $98 \%$ do seu valor em regime permanente $\left(\Delta T_{s}\right)$, podem ser estimados.

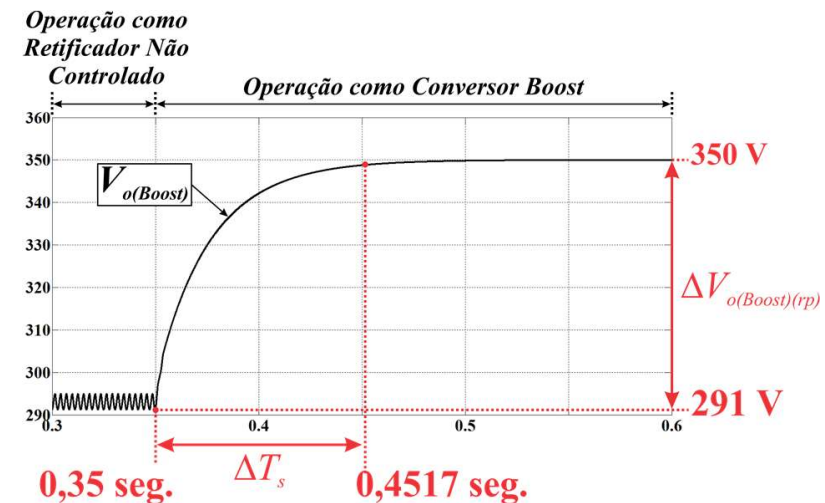

Fig. 8. Forma de onda da resposta de $V_{o(B o o s t)}$ ao degrau de amplitude das referências de corrente.

Assim, considera-se a obtenção da função de transferência do sistema equivalente do conversor Boost operando em malha aberta a partir de:

$$
G_{v i(e q)}(s)=K \frac{a}{s+a}
$$

sendo:

$$
\begin{gathered}
a=\frac{4}{\Delta T_{s}} \\
K=\frac{\Delta V_{o(\text { Boost })(r p)}}{\Delta A m p}
\end{gathered}
$$

onde $a$ é a frequência do polo do sistema de primeira ordem. 
Com base em (1), a função de transferência do sistema em malha aberta do conversor Boost para a condição normal de suprimento da rede CA trifásica de alimentação é definida por:

$$
G_{v i(e q)}(s)=11,683 \frac{39,33}{s+39,33}
$$

De posse da função de transferência $G_{v i(e q)}(s)$, fechou-se a malha do sistema e projetou-se um controlador de tensão que permita que o sistema em malha fechada do conversor Boost opere com erro em regime permanente nulo; ultrapassagem percentual inferior a $2 \%$; margem de fase superior a $60^{\circ}$; tempo de acomodação reduzido o suficiente para que em condições de afundamentos temporários de tensão, a tensão de saída do conversor Boost não reduza a um valor inferior a $10 \%$ do valor determinado pela referência de tensão estabelecida em $350 \mathrm{~V}$; e frequência de passagem por $0 \mathrm{~dB}$ inferior a no mínimo um décimo da frequência de chaveamento do conversor, objetivando a atenuação de ruídos em alta frequência.

Assim, após análise do sistema em malha fechada, projetouse o controlador de tensão do conversor Boost cuja função de transferência é definida por:

$$
G_{v(B)}(s)=50 \frac{(s+39,33)}{s(s+250)} .
$$

Percebe-se através da resposta em frequência e da resposta ao degrau para o sistema em malha fechada compensado apresentados na Figura 9 que o controlador de tensão projetado permitiu que o sistema em malha fechada apresentasse um erro em regime permanente nulo; uma margem de fase de $70,9^{\circ}$, garantindo uma resposta mais amortecida e estável; e uma frequência de passagem por $0 \mathrm{~dB}$ baixa, definindo um sistema capaz de atenuar os ruídos de alta frequência provenientes do chaveamento dos interruptores.

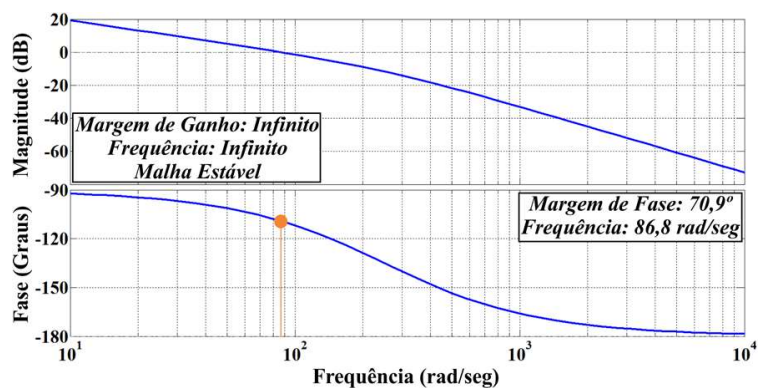

(a)

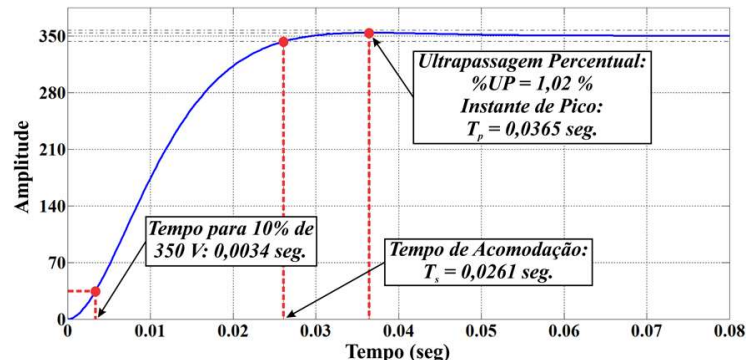

(b)

Fig. 9. Sistema do conversor Boost em malha fechada compensado: (a) diagramas de Bode e (b) resposta ao degrau.

\section{B. Projeto do Controlador de Tensão do Conversor Full-} Bridge

Para a solução do modelo matemático e o consequente projeto do controlador de tensão do conversor Full-Bridge considerou-se o circuito equivalente apresentado na Figura 10 Como sistema de análise, onde $R_{L n c}$ é a resistência dos enrolamentos de $L_{N C}, R_{L f b}$ é a resistência do enrolamento de $L_{F B}$ e $R_{p}, R_{s}$ e $R_{t}$ são as resistências dos enrolamentos do transformador.

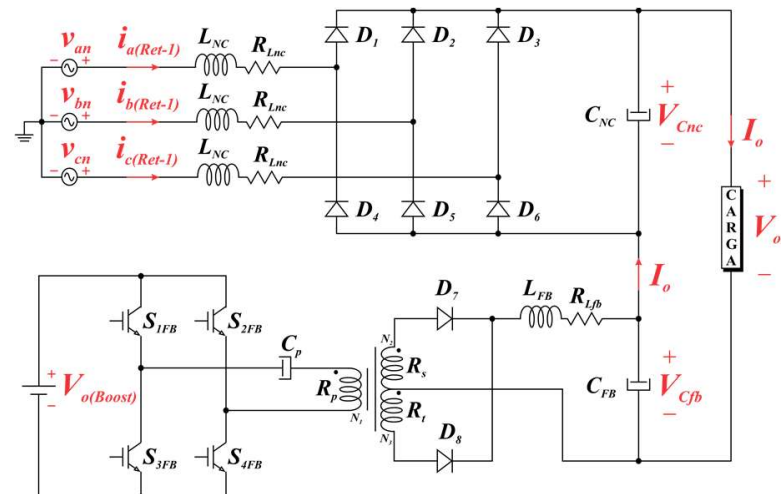

Fig. 10. Arranjo topológico equivalente da composição entre o retificador não controlado e o conversor Full-Bridge.

(2)

A Figura 11 apresenta o diagrama de blocos do sistema equivalente para a composição entre o RNC e o conversor FullBridge em malha fechada apresentando o controlador de tensão.

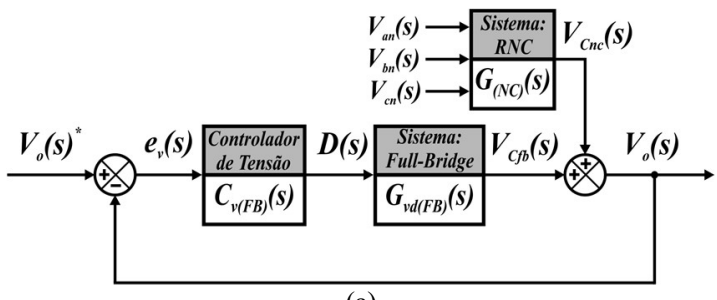

(a)

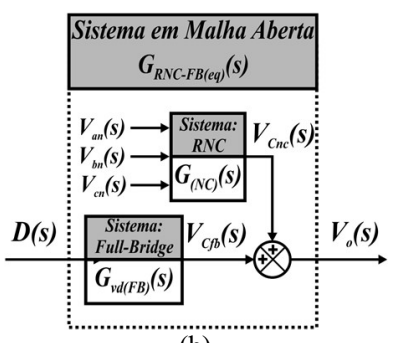

(b)

Fig. 11. Diagrama de blocos do sistema do circuito equivalente para a composição entre o RNC e o conversor Full-Bridge: (a) em malha fechada e (b) em malha aberta.

Para o estabelecimento da solução do modelo matemático do conversor Full-Bridge, foi necessário primeiramente definir quais são as variáveis manipulada e controlada do sistema. Segundo o que foi definido para estratégia de controle e conforme pode ser observado no diagrama de blocos do sistema equivalente para a composição entre o RNC e o conversor Full-Bridge em malha fechada apresentado na Figura 11(a), o controlador de tensão é responsável por interpretar o sinal de erro $\left(e_{v}\right)$ entre a referência de tensão $\left(V_{o}^{*}\right)$ e a tensão no barramento $\mathrm{CC}\left(V_{o}\right)$, o que permite atuar no 
sistema através da variação da razão cíclica de operação dos interruptores do circuito de potência do conversor Full-Bridge (D). Desta forma, o objetivo do controle do conversor FullBridge é manipular a razão cíclica de operação dos interruptores para controlar a tensão $V_{o}$, que por sua vez é definida pela soma das tensões $V_{C f b}$ e $V_{C n c}$.

Levando-se em consideração a amplitude unitária da onda dente-de-serra utilizada no controle PWM e o ganho unitário do sensor de tensão, o sistema em pauta é representado através de uma função de transferência $G_{R N C_{-} F B(e q)}(s)$ que relaciona a tensão regulada no barramento $\bar{C} C\left(V_{o}(s)\right.$ - variável controlada) com a variação da razão cíclica de operação dos interruptores do circuito do conversor Full-Bridge $(D(s)$ variável manipulada), conforme pode ser visualizado na Figura 11(b).

Segundo a solução do modelo matemático, para uma razão cíclica de 0,1845 ( $\triangle D)$, o conversor Full-Bridge permite a regulação da tensão no barramento CC em 400 V. A Figura 12 ilustra os comportamentos da resposta transitória da tensão no barramento CC no instante da execução do degrau de razão cíclica, onde é possível perceber que a resposta apresenta uma característica de respostas de sistemas de segunda ordem superamortecidos ou criticamente amortecidos, podendo ser aproximada em uma resposta de sistemas de primeira ordem com pequeno atraso de tempo (tempo morto).

Através da Figura 12, pôde-se também obter as informações necessárias para a estimativa da função de transferência do sistema equivalente da Figura $10, G_{R N C-F B(e q)}(s)$, tais como o valor em regime permanente da tensão $V_{o}$ após o degrau $\left(\Delta V_{o(r p)}\right)$; o tempo que a resposta da tensão no barramento leva para começar a responder ao degrau, também chamado de atraso de transporte $(\Delta \theta)$; e o tempo necessário para a resposta da tensão $V_{o}$ atingir $98 \%$ do seu valor em regime permanente $\left(\Delta T_{s}\right)$ após o atraso de transporte.

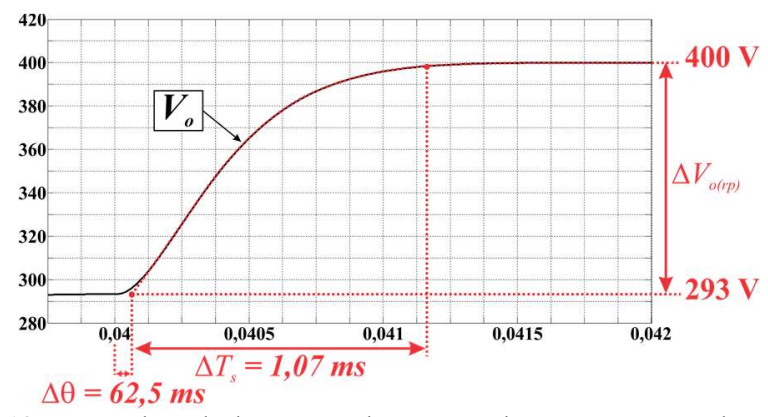

Fig. 12. Forma de onda da resposta da tensão no barramento CC ao degrau de razão cíclica.

Assim, o comportamento do sistema do circuito equivalente da Figura 10 pode ser representado através de uma função de transferência de primeira ordem com tempo morto conforme definido por:

$$
G_{R N C-F B(e q)}(s)=K \frac{a}{s+a} e^{-\Delta \theta s}
$$

sendo:

$$
a=\frac{4}{\Delta T_{s}}
$$

$$
K=\frac{\Delta V_{o(r p)}}{\Delta D}
$$

onde $a$ é a frequência do polo do sistema de primeira ordem e $e^{-\Delta \theta s}$ representa a transformada de Laplace para o atraso no tempo.

Para valores pequenos de atraso de transporte [24], é possível substituir $e^{-\Delta \theta s}$ pela aproximação de primeira ordem utilizando a série de Taylor para funções exponenciais [25]:

$$
\begin{aligned}
e^{-\Delta \theta s}=\frac{1}{e^{\Delta \theta s}}= & \frac{1}{1+\Delta \theta s+\frac{(\Delta \theta s)^{2}}{2}+\frac{(\Delta \theta s)^{3}}{6}+\cdots} \\
& \approx \frac{1}{1+\Delta \theta s} .
\end{aligned}
$$

Substituindo a aproximação representada por (9) em (6), tem-se que:

$$
G_{R N C-F B(e q)}(s)=K \frac{a}{(s+a)(1+\Delta \theta s)} .
$$

Com base em (10), a função de transferência do sistema em malha aberta do circuito equivalente para a composição entre o RNC e o conversor Full-Bridge para condições normais de suprimento da rede CA trifásica de alimentação é definida por:

$$
\begin{aligned}
& G_{R N C-F B(e q)}(s) \\
& =\frac{34688346883,5}{s^{2}+19738,317757 s+59813084,1122}
\end{aligned}
$$

Destaca-se que o transformador do conversor Full-Bridge foi construído por uma empresa especializada que proporcionou uma elevada indutância magnetizante e reduzida indutância de dispersão. Estes parâmetros não foram considerados em (11) e não influenciaram de forma efetiva na dinâmica do controle do conversor, conforme pode ser verificado nas formas de onda da tensão de saída apresentadas nos resultados experimentais (Seção V).

Para as mesmas especificações de projeto apresentadas para o conversor Boost, projetou-se o controlador de tensão do conversor Full-Bridge cuja função de transferência é definida por:

$$
C_{v(F B)}(s)=0,008 \frac{(s+1000)}{s} .
$$

A resposta em frequência e a resposta ao degrau para o sistema em malha fechada compensado são apresentados na Figura 13. Conforme pode ser observado, o controlador de tensão projetado atendeu aos requisitos para o projeto do sistema em malha fechada compensado, onde o erro em regime permanente da resposta ao degrau manteve-se nulo; a margem de fase do sistema manteve-se em $62,6^{\circ}$; a ultrapassagem percentual apresentou-se em $0,308 \%$; e a frequência de passagem por $0 \mathrm{~dB}$ apresentou o valor inferior a um décimo da frequência angular de chaveamento. 


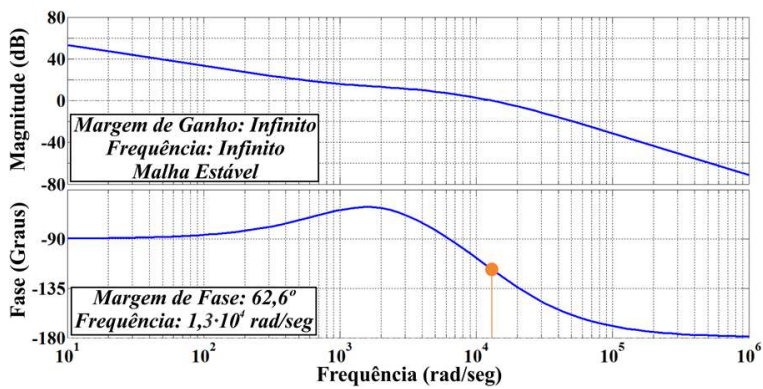

(a)

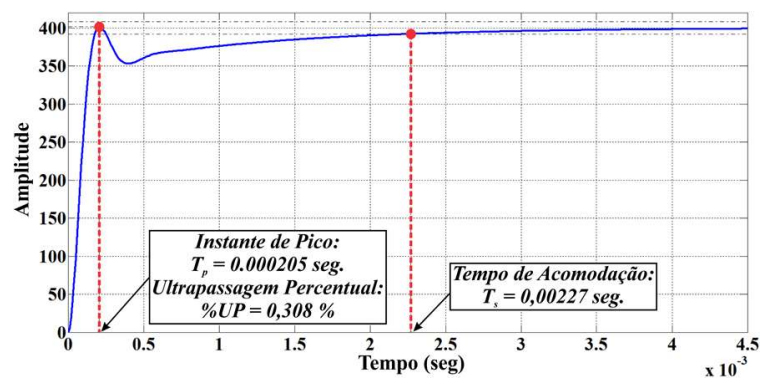

(b)

Fig. 13. Sistema para a composição entre o RNC e o conversor Full-Bridge em malha fechada compensado: (a) diagramas de Bode e (b) resposta ao degrau.

\section{RESULTADOS EXPERIMENTAIS}

Um protótipo do retificador híbrido trifásico proposto foi montado e analisado em laboratório, como pode ser visualizado na Figura 14. As especificações de projeto e os parâmetros do circuito de potência são apresentados nas Tabelas I e II, respectivamente.

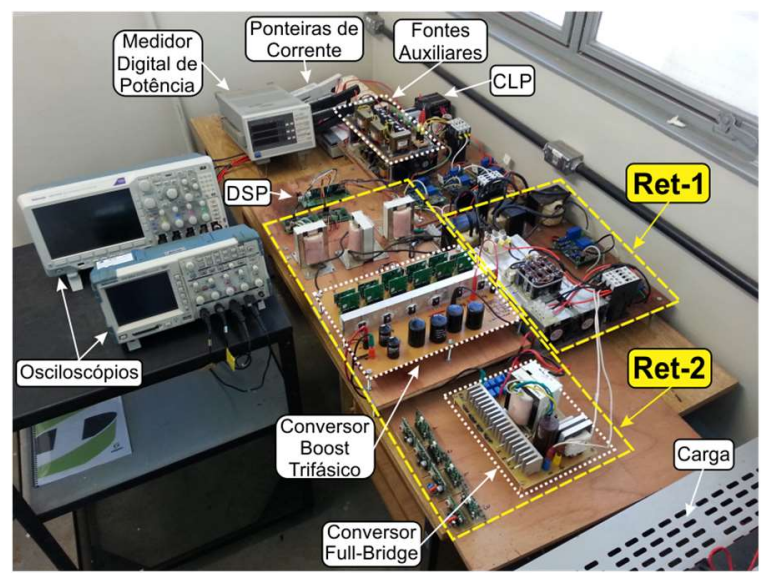

Fig. 14. Protótipo do retificador híbrido trifásico desenvolvido em laboratório.

\section{A. Resultados Experimentais sob Condições Normais de Suprimento da Rede CA}

Para validar a eficácia da estratégia de controle do ponto de vista de imposição de correntes de linha de entrada senoidais, a Figura 15 apresenta as correntes de linha de entrada das fases $A, B$ e $C$ e a Figura 16 demonstra como é realizada a composição das correntes drenadas pela fase $A$ dos grupos retificadores Ret-1, Ret-2 e do RHT. O conteúdo harmônico das correntes pode ser observado pela Figura 17.

Ensaios experimentais envolvendo variações de carga foram realizados e determinou-se a distorção harmônica total média entre as correntes impostas e o fator de potência médio para ampla faixa de variação de carga, conforme observado na Figura 18. A Figura 19 demonstra as tensões nos capacitores de saída de Ret-1 $\left(V_{C n c}\right)$, de Ret-2 $\left(V_{C f b}\right)$ e do conversor Boost $\left(V_{o(B o o s t}\right)$, e a tensão no barramento CC $\left(V_{o}\right)$ controlada em 400 $\mathrm{V}$ durante condições normais de suprimento da rede elétrica. Observa-se que, conforme esperado, o controle promove a compensação série de tensão no barramento $\mathrm{CC}$ e a tensão total aplicada à carga é o resultado da soma entre $V_{C n c}$ e $V_{C f b}$.

Através da utilização do medidor digital de potência, mediuse a potência ativa média total processada por cada grupo retificador durante condições normais de suprimento da rede CA e uma carga com potência de aproximadamente $4,5 \mathrm{~kW}$, conforme pode ser verificador pela Figura 20. Assim como foi previsto, a estrutura Ret-1 é responsável por mais de 73\% da potência ativa média total processada pelo RHT, restando menos de $27 \%$ para a estrutura Ret-2, o que determina uma maior robustez e confiabilidade operacional para a estrutura global do RHT.

\section{TABELA I}

Especificações de Projeto do Retificador Híbrido Trifásico Implementado

\begin{tabular}{|c|c|c|}
\hline Parâmetro & Símbolo & Valor \\
\hline $\begin{array}{c}\text { Frequência máxima de chaveamento dos } \\
\text { conversor Boost e Full-Bridge }\end{array}$ & $f_{c h}$ & $25 \mathrm{kHz}$ \\
\hline $\begin{array}{c}\text { Potência média processada por Ret-2 para a } \\
\text { condição de 50\% de afundamento }\end{array}$ & $P_{o(\text { Ret-2) }}$ & $3,17 \mathrm{~kW}$ \\
\hline Potência media total de saída & $P_{o}$ & $5 \mathrm{~kW}$ \\
\hline $\begin{array}{c}\text { Razão cíclica máxima permitida de operação do } \\
\text { conversor Full-Bridge }\end{array}$ & $d_{m a x}$ & 0,49 \\
\hline $\begin{array}{c}\text { Relação de expiras entre primário e secundário } \\
\text { do transformador do conversor Full-Bridge }\end{array}$ & $N_{l} / N_{2}=$ & $N_{l} / N_{3}$ \\
\hline Tensão média de saída do conversor Boost & $V_{o(B o o s t)}$ & $350 \mathrm{~V}$ \\
\hline $\begin{array}{c}\text { Tensão média de saída do conversor Full-Bridge } \\
\text { para a razão cíclica máxima }\end{array}$ & $V_{C f b}$ & $343 \mathrm{~V}$ \\
\hline $\begin{array}{c}\text { Tensão média no barramento CC } \\
\text { Tensão média no capacitor } C_{N C}\end{array}$ & $V_{o}$ & $400 \mathrm{~V}$ \\
\hline $\begin{array}{c}\text { Valor de pico das tensões } v_{a n}, v_{b n} \text { e } v_{c n} \text { para } \\
\text { condição normal de suprimento da rede }\end{array}$ & $V_{p}$ & $180 \mathrm{~V}$ \\
\hline
\end{tabular}

TABELA II

Parâmetros do Protótipo do Retificador Híbrido Trifásico Implementado

\begin{tabular}{|c|c|c|c|}
\hline \multirow{2}{*}{ Componentes } & \multirow{2}{*}{ Ret-1 } & \multicolumn{2}{|c|}{ Ret-2 } \\
\hline & & Boost & Full-Bridge \\
\hline $\begin{array}{c}\text { Ponte } \\
\text { Retificadora } \\
\text { Não } \\
\text { Controlada }\end{array}$ & $\begin{array}{l}\text { Modelo } \\
\text { SKD 62/12 da } \\
\text { SEMIKRON }^{\circledR}\end{array}$ & - & - \\
\hline Indutores & $\begin{array}{c}3 \text { Indutores } \\
L_{N C}=900 \mu \mathrm{H}\end{array}$ & $\begin{array}{l}3 \text { Indutores } \\
L_{B}=2 \mathrm{mH}\end{array}$ & $L_{F B}=200 \mu \mathrm{H}$ \\
\hline \multirow{2}{*}{ Capacitores } & \multirow{2}{*}{$C_{N C}=3150 \mu \mathrm{F}$} & \multirow{2}{*}{$C_{B}=600 \mu \mathrm{F}$} & $C_{p}=3,3 \mu \mathrm{F}$ \\
\hline & & & $C_{F B}=270 \mu \mathrm{F}$ \\
\hline $\begin{array}{c}\text { Ponte } \\
\text { Retificadora } \\
\text { Controlada }\end{array}$ & - & $\begin{array}{l}6 \text { Interruptores } \\
\text { Modelo } \\
\text { C2M0080120D } \\
\text { da CREE }^{\circledR}\end{array}$ & - \\
\hline $\begin{array}{c}\text { Ponte } \\
\text { Inversora }\end{array}$ & - & - & $\begin{array}{l}4 \text { Interruptores } \\
\text { Modelo } \\
\text { C2M0080120D } \\
\text { da CREE }^{\circledR}\end{array}$ \\
\hline Diodo & - & - & $\begin{array}{c}3 \text { diodos } \\
\text { Modelo } \\
\text { C3D10065 da } \\
\text { CREE }^{\circledR}\end{array}$ \\
\hline
\end{tabular}




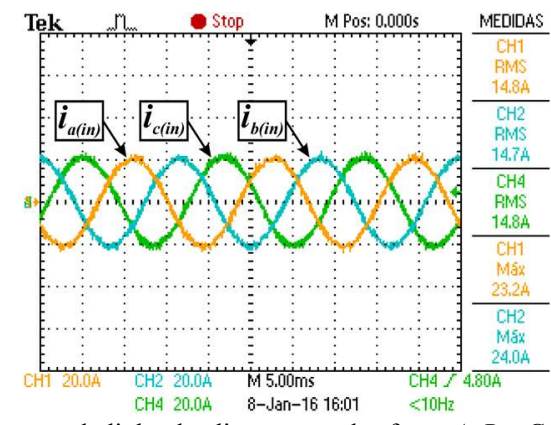

Fig. 15. Correntes de linha de alimentação das fases $A, B$ e $C$.

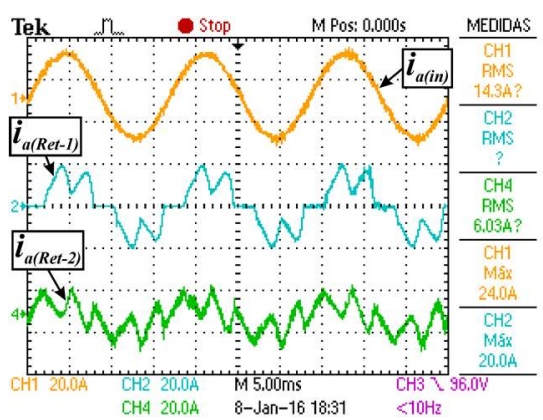

Fig. 16. Fase $A$ : Correntes de linha de entrada drenadas pelo RHT, por Ret-1 e por Ret-2.

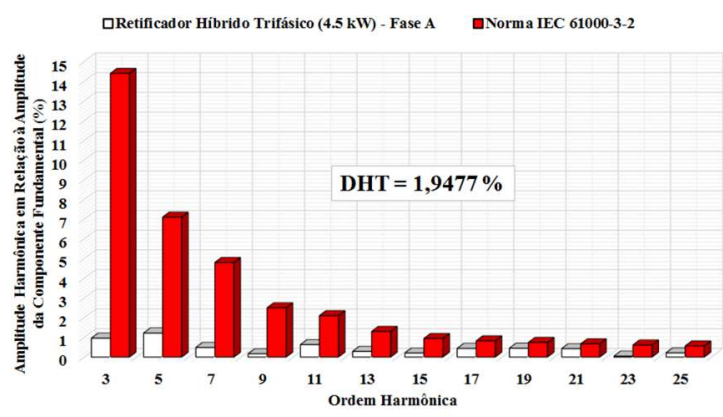

(a)

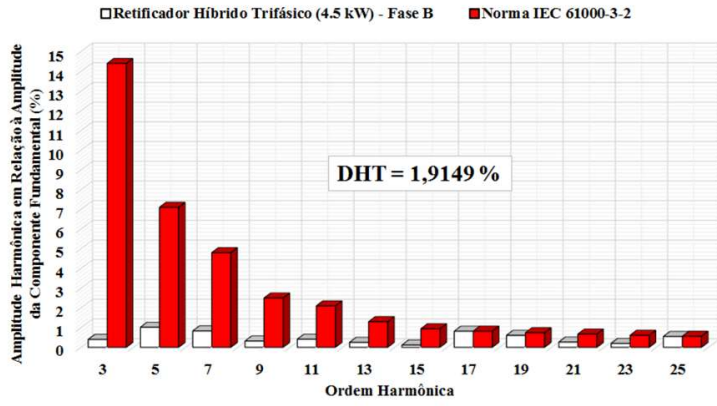

(b)

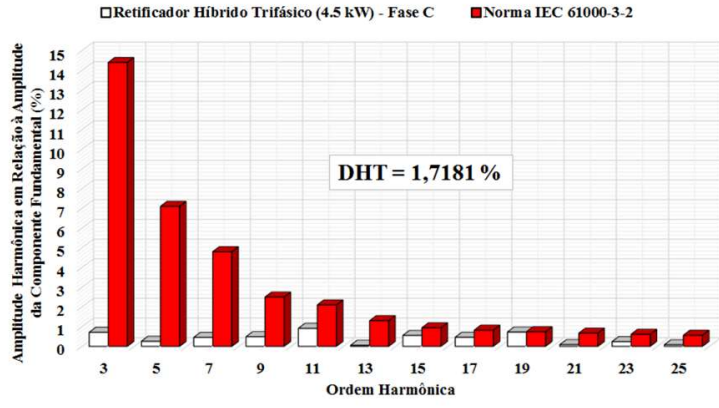

(c)

Fig. 17. Distorção harmônica total e comparação, em relação à normal IEC 61000-3-2, das amplitudes harmônicas das correntes (a) $i_{a(i n)}$, (b) $i_{b(i n)}$ e (c) $i_{c(i n)}$.

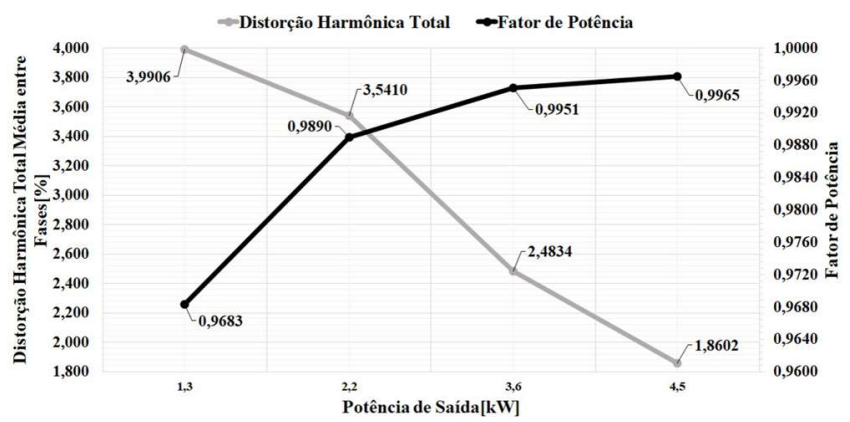

Fig. 18. Distorção harmônica total média e fator de potência médio em função da potência ativa média total processada.

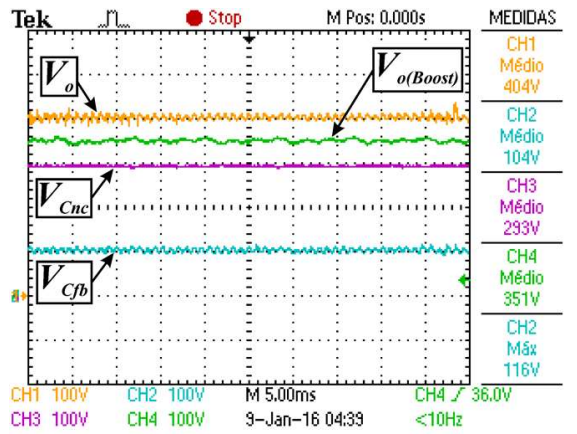

Fig. 19. Tensão na carga $\left(V_{o}\right)$, tensão de saída de Ret-1 $\left(V_{C n c}\right)$, e tensão de saída de Ret-2 $\left(V_{C f b}\right)$.

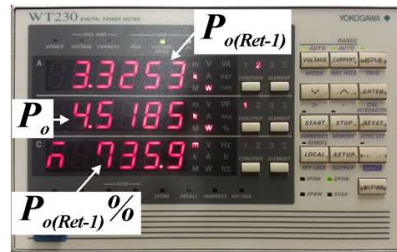

(a)

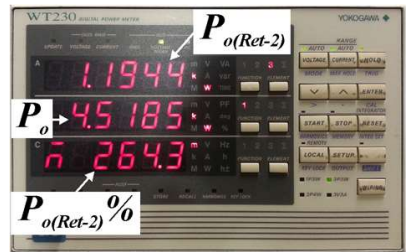

(b)
Fig. 20. Potências ativas de saída processadas pelo retificador híbrido trifásico $\left(P_{o}\right)$, por Ret-1 $\left(P_{o(\text { Ret-1 } 1)}\right)$ e por Ret-2 $\left(P_{o(\text { Ret-2 })}\right)$ e percental de processamento de potência $\left(P_{o(\text { Ret- }-1)} \%\right.$ e $\left.P_{o(\text { Ret }-2)} \%\right)$.

\section{B. Resultados Experimentais do RHT Durante Degrau de Carga}

As correntes de linha de entrada e as correntes drenadas por Ret-1 e Ret-2 para um degrau de carga de $2,4 \mathrm{~kW}$ para $4,5 \mathrm{~kW}$ são apresentados nas Figuras 21(a) e (b), respectivamente. $\mathrm{O}$ comportamento da tensão no barramento $\mathrm{CC}$ e das tensões nos capacitores de saída de Ret-1, Ret-2 e do conversor Boost durante o transitório de carga também foram verificados e são apresentados na Figura 21(c). A tensão $V_{C n c}$ apresentou uma pequena redução após o degrau, entretanto a tensão $V_{o}$ se manteve constante em $400 \mathrm{~V}$.

Em termos de aplicações voltadas para microrredes, as respostas das tensões $V_{C f b}$ e $V_{o}$ diante de um degrau de carga ilustram o comportamento esperado quando da ocorrência de reduções de fornecimento de energia por parte de fontes renováveis de energia e de conexões de cargas de alta potência em barramentos de distribuição de energia em CC. Assim, percebe-se que o RHT é uma excelente alternativa para promover suportabilidade a oscilações de tensão em barramentos $\mathrm{CC}$ de microrredes. 


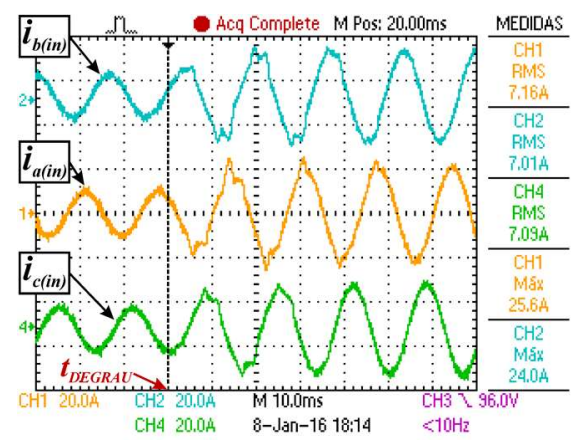

(a)

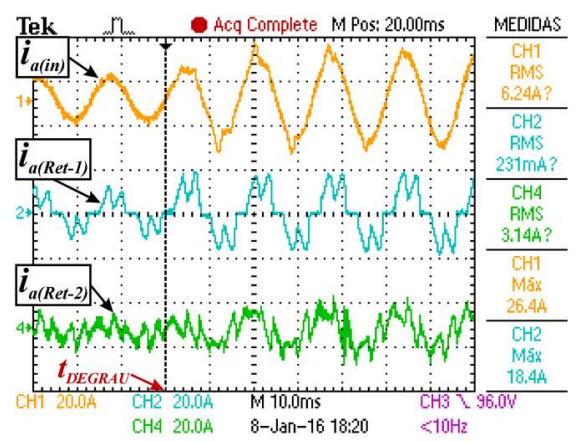

(b)

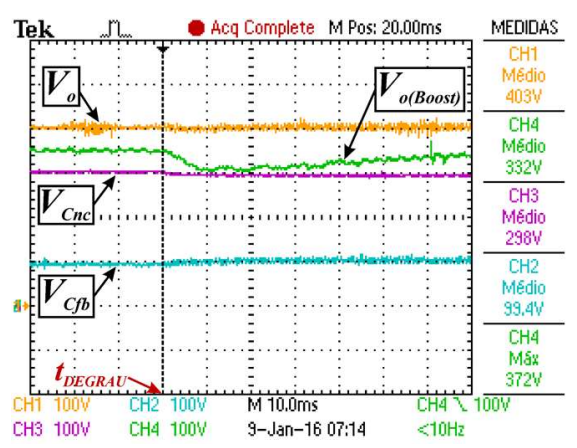

(c)

Fig. 21. Respostas dinâmicas durante um degrau de carga de 2,4 kW para 4,5 kW: (a) Correntes de linha de entrada, (b) correntes de linha de entrada drenadas pela fase $A$ do RHT, de Ret-1 e de Ret-2 e (c) tensões no barramento CC, nos capacitores de saída de Ret-1, de Ret-2 e do conversor Boost

\section{Resultados Experimentais do RHT Operando sob} Condições de Afundamentos de Tensão

Para os testes envolvendo afundamentos de tensão utilizouse a fonte trifásica programável FCATHQ 450-22-100 da SUPPLIER ${ }^{\circledR}$. Durante estes testes foi necessário reduzir a potência da carga de $4,5 \mathrm{~kW}$ para $1,3 \mathrm{~kW}$ visto às limitações de corrente da fonte programável utilizada.

A Figura 22 ilustra as formas de onda das tensões de fase; das correntes de linha de entrada; e das tensões no barramento CC, na saída de Ret-1 e na saída de Ret-2, durante a ocorrência de um afundamento do Tipo $A$ (com variação de 50\% nas amplitudes das tensões de alimentação). Analogamente, as Figuras 23 a 28 ilustram as principais formas de onda obtidas durante a ocorrência de afundamentos do Tipo $B, C, D, E, F$ e $G$. Nota-se que a estratégia de controle se demonstrou eficaz fornecendo um barramento CC com tensão constante de $400 \mathrm{~V}$ às cargas. No que tange o controle de corrente, a imposição de correntes de linha de entrada senoidais é realizada mesmo durante a ocorrência de afundamentos de tensão assimétricos que implicam na variação do ângulo de fase das tensões de alimentação.

As Figuras 29 e 30 demonstram a divisão de processamento de potência entre Ret-1 e Ret-2 e o fator de potência por fase, respectivamente, em função da potência total entregue à carga e em função do tipo de afundamento temporário de tensão ao qual o RHT foi submetido. Observa-se que, apesar do processamento de potência por parte de Ret-1 cair em decorrência da diminuição da amplitude das tensões de fase, Ret-2 consegue suprir a potência adicional entregue à carga de tal forma que a potência ativa total de saída permaneça constante em $4,5 \mathrm{~kW}$. A Figura 30 permite verificar que mesmo diante da variação no ângulo de fase das tensões F-N provocada pelos afundamentos dos tipos $\mathrm{C}, \mathrm{D}, \mathrm{F}$ e $\mathrm{G}$, o fator de potência por fase demonstrou-se elevado.

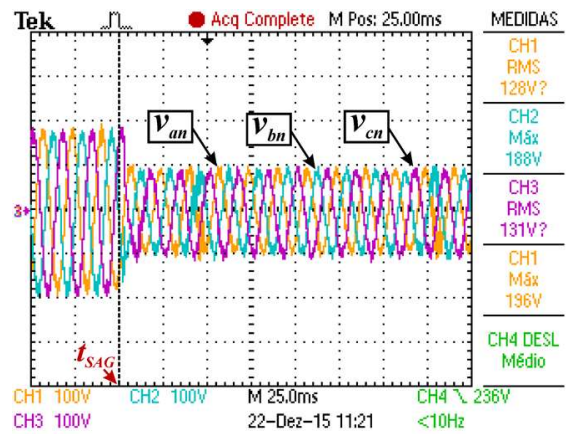

(a)

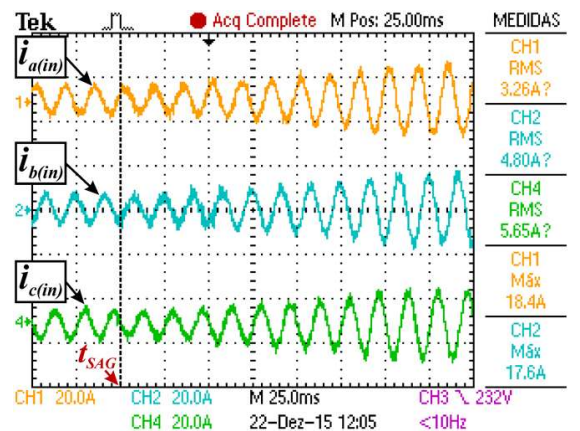

(b)

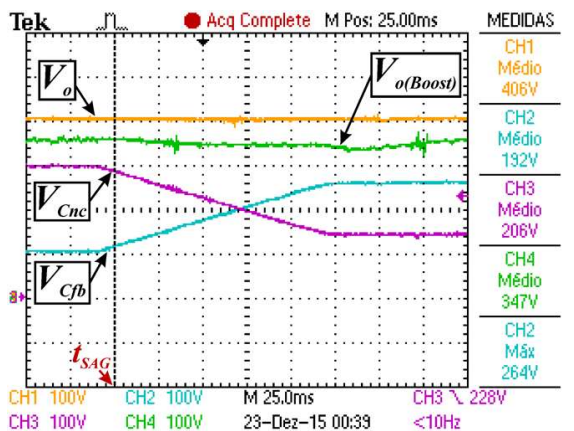

(c)

Fig. 22. (a) Tensões de fase; (b) correntes $i_{a(i n)}, i_{b(i n)}$ e $i_{c(i n)}$; e (c) tensões $V_{o}, V_{o(B o s s t)}, V_{C n c}$ e $V_{C f b}$ durante um afundamento do Tipo $A$.

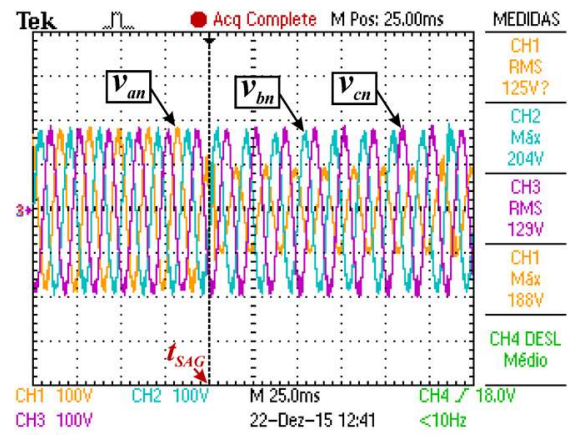

(a)

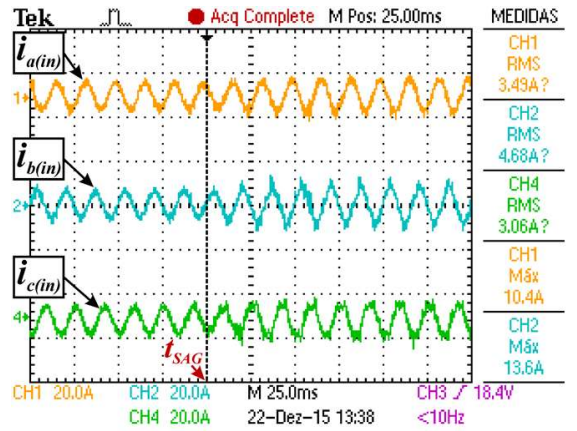

(b)

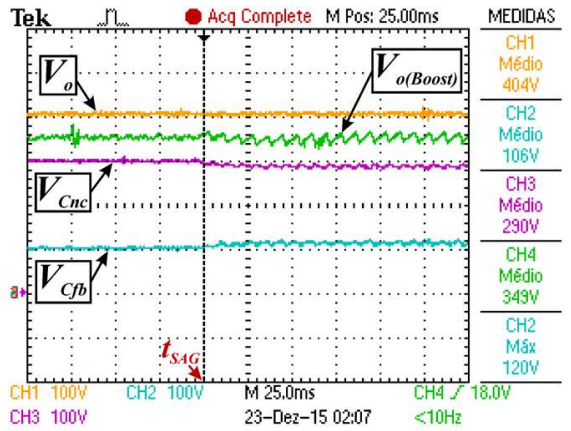

(c)

Fig. 23. (a) Tensões de fase; (b) correntes $i_{a(i n)}, i_{b(i n)}$ e $i_{c(i n)}$; e (c) tensões $V_{o}, V_{o(B o o s t)}, V_{C n c}$ e $V_{C \zeta b}$ durante um afundamento do Tipo $B$ 


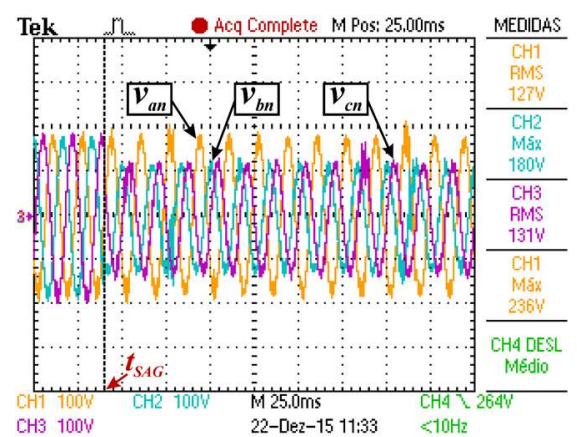

(a)

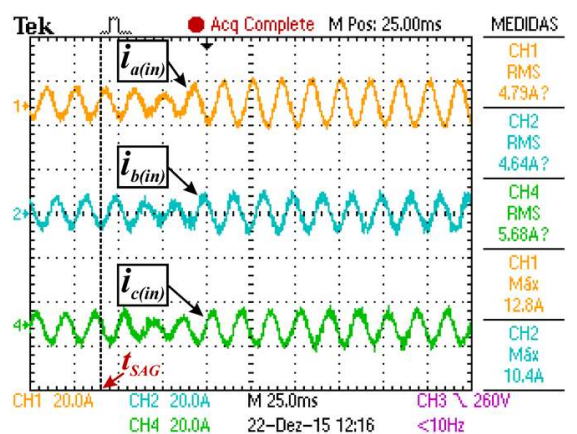

(b)

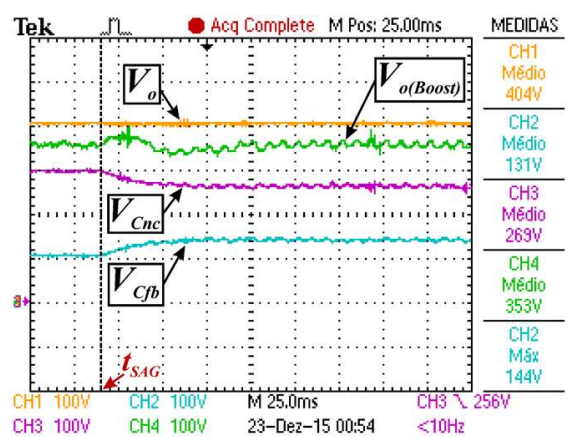

(c)

Fig. 24. (a) Tensões de fase; (b) correntes $i_{a(i n)}, i_{b(i n)}$ e $i_{c(i n)}$; e (c) tensões $V_{o}, V_{o(B o o s t)}, V_{C n c}$ e $V_{C f b}$ durante um afundamento do Tipo $C$.

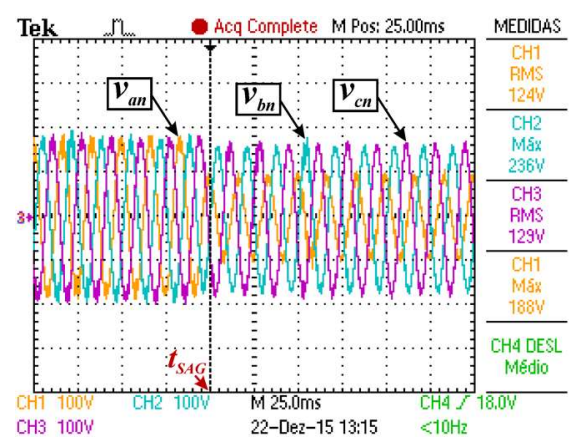

(a)

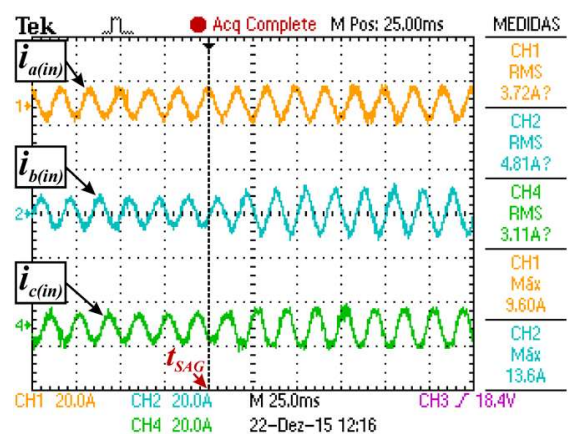

(b)

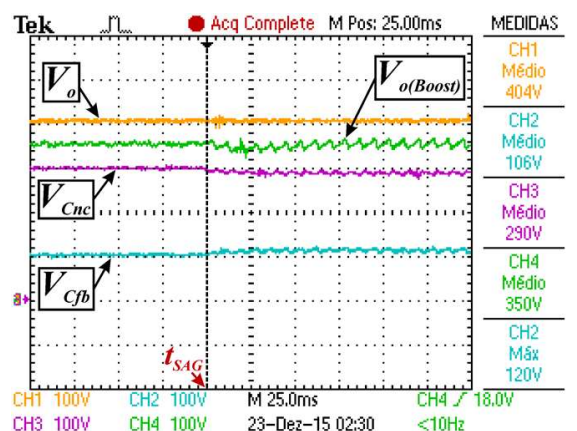

(c)

Fig. 25. (a) Tensões de fase; (b) correntes $i_{a(i n)}, i_{b(i n)}$ e $i_{c(i n)}$; e (c) tensões $V_{o}, V_{o(B o s s t)}, V_{C n c}$ e $V_{C f b}$ durante um afundamento do Tipo D.

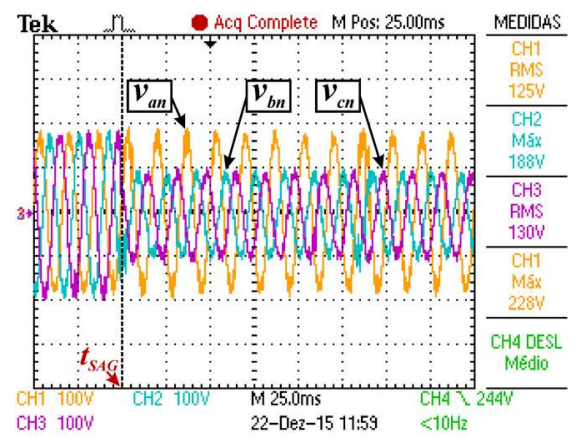

(a)

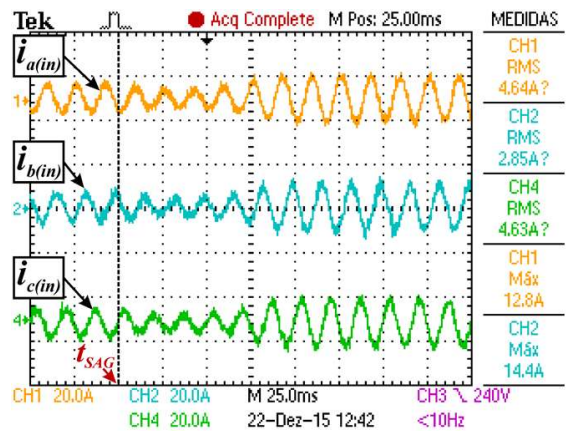

(b)

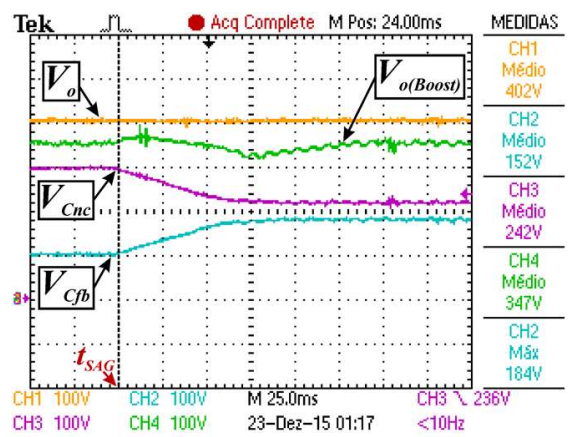

(c)

Fig. 26. (a) Tensões de fase; (b) correntes $i_{a(i n)}, i_{b(i n)}$ e $i_{c(i n)}$; e (c) tensões $V_{o}, V_{o(B o o s t)}, V_{C n c}$ e $V_{C f b}$ durante um afundamento do Tipo E.

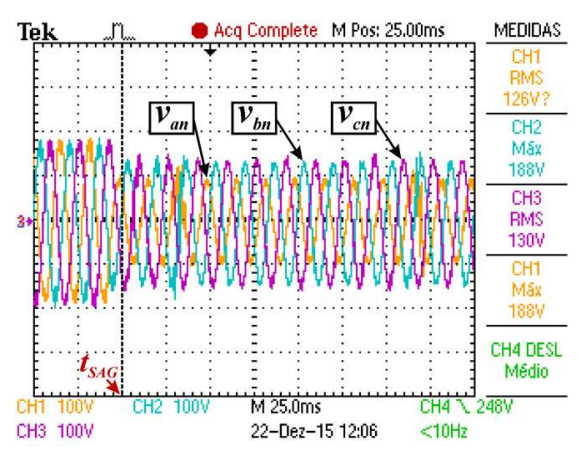

(a)

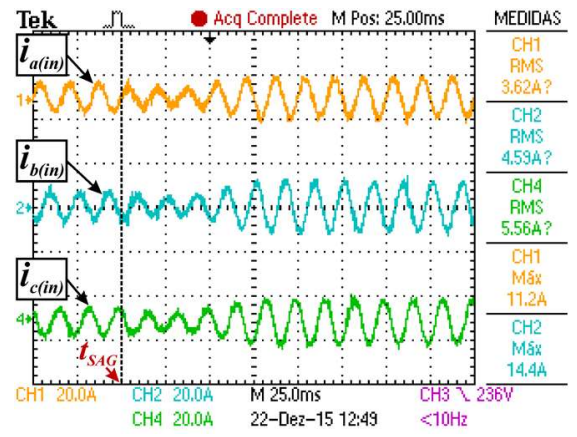

(b)

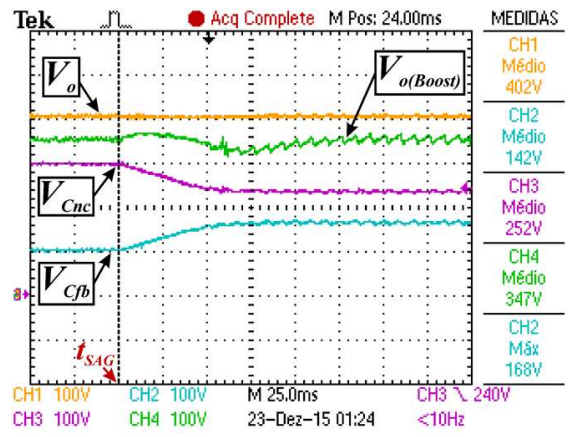

(c)

Fig. 27. (a) Tensões de fase; (b) correntes $i_{a(i n)}, i_{b(i n)}$ e $i_{c(i n)}$; e (c) tensões $V_{o}, V_{o(B o o s t)}, V_{C n c}$ e $V_{C f b}$ durante um afundamento do Tipo $F$. 


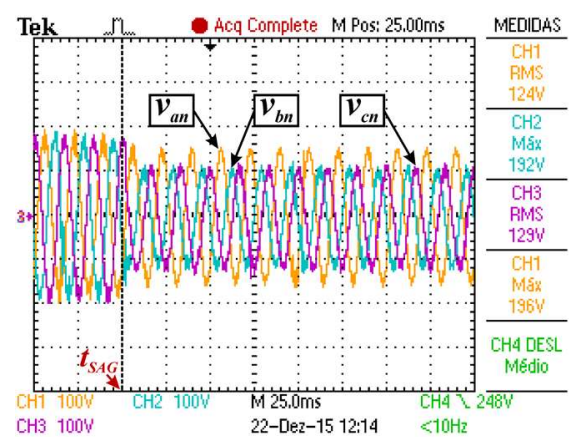

(a)

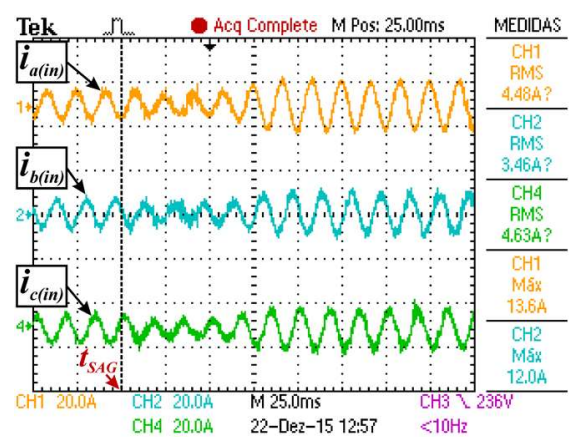

(b)

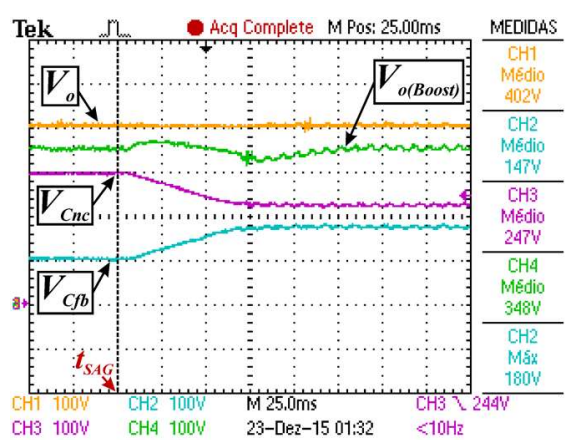

(c)

Fig. 28. (a) Tensões de fase; (b) correntes $i_{a(i n)}, i_{b(i n)}$ e $i_{c(i n)}$; e (c) tensões $V_{o}, V_{o(B o o s t)}, V_{C n c}$ e $V_{C f b}$ durante um afundamento do Tipo $G$.

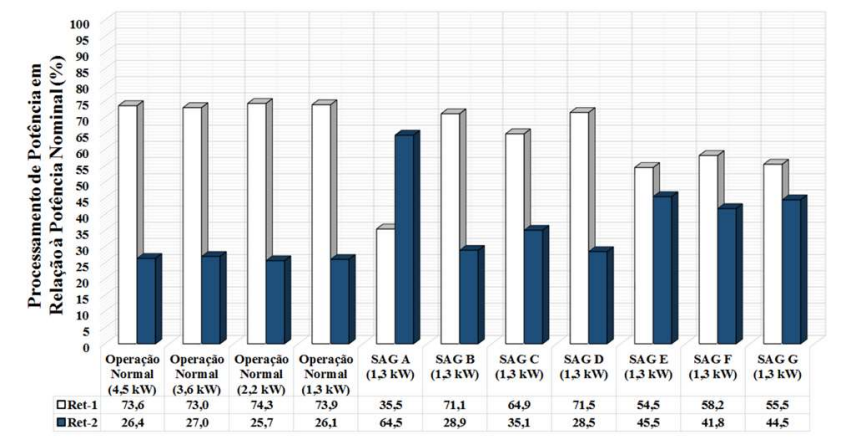

Fig. 29. Divisão de processamento de potência entre Ret-1 e Ret-2 em função da potência total de saída e do tipo de afundamento de tensão.

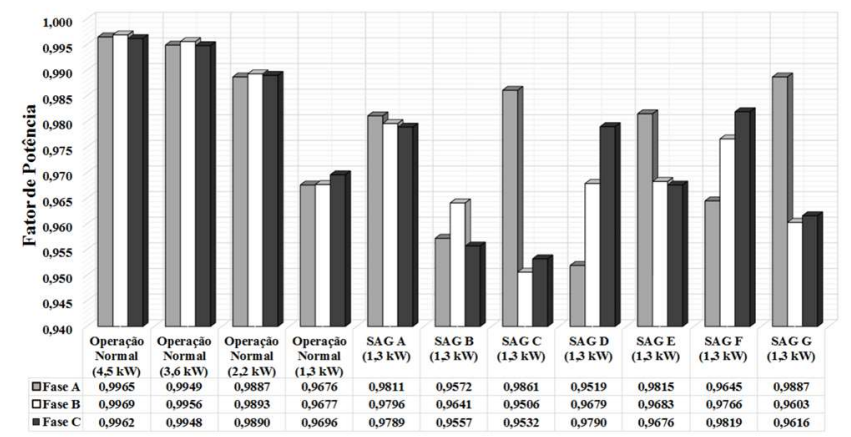

Fig. 30. Fator de potência por fase em função da potência total de saída e do tipo de afundamento de tensão.

\section{CONCLUSÃO}

Este artigo propõe a utilização de um conversor CA-CC híbrido. Como nova contribuição, foram apresentadas as principais características de uma nova estrutura topológica de retificador híbrido trifásico que é capaz de promover imposição de correntes de linha de entrada senoidais e compensação série de tensão no barramento CC, promovendo assim, suportabilidade aos afundamentos temporários de tensão tanto na lado CA quanto no lado CC.

Como método de validação da topologia proposta, foram traçados os detalhes operacionais, incluindo as descrições do princípio de funcionamento, da estratégia de controle e a demonstração dos principais resultados experimentais.

No que tange seu potencial para aplicação em microrredes $\mathrm{CC}$ conectadas à rede de distribuição em CA, ressalta-se a excelente suportabilidade à afundamentos de tensão diante das intermitências inerentes às fontes de energias renováveis como a solar e a eólica - e aos severos transitórios de cargas.
A fim de apresentar uma solução que torne possível a injeção de potência ativa na rede CA utilizando o retificador híbrido proposto, novos estudos estão sendo realizados no que tange ao projeto de um conversor Full-Bridge bidirecional. Os resultados obtidos serão reportados em breve em um novo trabalho atualmente em desenvolvimento.

\section{AGRADECIMENTOS}

Os autores agradecem ao CNPq (processos 141567/2013-9, 474055/2013-2 e 304307/2013-0), à FAPEMIG (processos APQ-00023-12 e TEC-PPM00031-14) e à CAPES pelo apoio financeiro.

\section{REFERÊNCIAS}

[1] D. Salomonsson, L. Söder, A. Sannino, "Protection of Low-Voltage DC Microgrids", IEEE Transactions on Power Delivery, Vol. 24, No. 3, pp. 1045-1053, July 2009.

[2] H. Kakigano, Y. Miura, T. Ise, "Low-Voltage BipolarType DC Microgrid for Super High Quality Distribution", IEEE Transactions on Power Electronics, Vol. 25, No. 12, pp. 3066-3075, Dec. 2010.

[3] P. C. Loh, D. Li, Y. K. Chai, F. Blaabjerg, "Hybrid ACDC Microgrids With Energy Storages and Progressive Energy Flow Tuning", in International Power Electronics and Motion Control Conference, Vol. 1, pp. 120-127, June 2012.

[4] M. Elshaer, A. Mohamed, O. Mohammed, "Grid Connected DC Distribution System for Efficient Integration of Sustainable Energy Sources", IEEE Power Systems Conference and Exposition, pp. 1-9, May 2011.

[5] D. M. Vilathgamuwa, P. C. Loh, Y. Li, "Protection of Microgrids During Utility Voltage Sags", IEEE Transactions on Industrial Electronics, Vol. 53, No. 5, pp. 1427-1436, Oct. 2006.

[6] D. Salomonsson, A. Sannino, "Low-Voltage DC Distribution System for Commercial Power Systems With Sensitive Electronic Loads", IEEE Transactions on Power Delivery, Vol. 22, No. 3, pp. 1620-1627, July 2007. 
[7] M. Imecs, C. Szabo, I. I. Incze, "Modelling and simulation of controlled bi-directional power electronic converters in a DC energy distribution line with AC grid- and motor-side active filtering", in European Conference on Power Electronics and Applications, pp. 1-10, Sept. 2007.

[8] P. K. Steimer, "Enabled by high power electronics Energy efficiency, renewables and smart grids", in International Power Electronics Conference (IPEC), pp. 11-15, June 2010.

[9] T. Wu, C. Chang, L. Lin, G. Yu, Y. Chang, "DC-Bus Voltage Control with a Three-Phase Bi-directional Inverter for DC Distribution Systems", IEEE Transactions on Power Electronics, Vol. 28, No. 4, pp. 1890-1899, Apr. 2013.

[10] B. Zhao, Q. Yu, W. Sun, "Extended-Phase-Shift Control of Isolated Bidirectional DC-DC Converter for Power Distribution in Microgrid", IEEE Transactions on Power Electronics, Vol. 27, No. 11, pp. 4667-4680, Nov. 2012.

[11] H. G. Sarmiento, E. Estrada, "A Voltage Sag Study in an Industry with Adjustable Speed Drives", IEEE Industry Applications Magazine, Vol. 2, No. 1, pp. 1619, Jan./Feb. 1996.

[12] M. H. J. Bollen, L. D. Zhang, "Analysis of Voltage Tolerance of AC Adjustable-speed Drives for Threephase Balanced and Unbalanced Sags", IEEE Transaction on Industrial Applications, Vol. 36, No. 3, pp. 904 - 910, May/June 2000.

[13] P. C. A. Leao, K. D. Rodrigues, J. C. de Oliveira, "Conversores de Frequência VSI-PWM Submetidos a Afundamentos Temporários de Tensão ("Voltage Sags")", Controle \& Automação, Vol. 14, No. 1, Jan./Mar. 2003.

[14] S. Z. Djokic, K. Stockman, J. V. Milanovic, J. J. M. Desmet, R. Belmans, "Sensitivity of AC Adjustable Speed Drives to Voltage Sags and Short Interruptions", IEEE Transaction on Power Delivery, Vol. 20, No. 1, pp. 494-505, Jan. 2005.

[15] M. H. J. Bollen, Understanding Power Quality Problems: Voltage Sags and Interruptions, IEEE Press, New York, 2000.

[16] C. J. O. Junior; L. P. Pires, L. C. Freitas, E. A. A. Coelho, G. B. Lima, L. C. G. Freitas, "Design, Analysis and Performance of a Bidirectional Solar Inverter with a Global and Independent Maximum Power Extraction Technique", IET Power Electronics, Vol. 11, No. 1, pp. 221-228, Feb. 2018

[17] D. B. Rodrigues, A. V. Costa, G. B. Lima, L. C. Freitas, E. A. A. Coelho, V. J. Farias, L. C. G. Freitas, "DSPbased Implementation of Control Strategy for Sinusoidal Input Line Current Imposition for a Hybrid Three-phase Rectifier", IEEE Transactions on Industrial Informatics, Vol. 9, No. 4, pp. 1947-1963, Nov. 2013.

[18] A. V. Costa, D. B. Rodrigues, G. B. Lima, L. C. Freitas, E. A. A. Coelho, V. J. Farias, L. C. G. Freitas, "New
Hybrid High-Power Rectifier With Reduced THDI and Voltage-Sag Ride-Through Capability Using Boost Converter", IEEE Transactions on Industry Applications, Vol. 49, No. 6, pp. 2421-2436, Nov./Dec. 2013.

[19] A.V. Costa, D.B. Rodrigues, G.B. Lima, L.C. Freitas, E. A. A. Coelho, V. J. Farias, L. C. G. Freitas, "Retrofitting Technique to Improve Voltage Sags RideThrough Capability of ASD Using SEPIC Rectifiers", in Proc. of IEEE Applied Power Electronics Conference and Exposition, pp. 1469-1476, Feb. 2012.

[20] A.V. Costa, D.B. Rodrigues, G.B. Lima, L.C. Freitas, E. A. A. Coelho, V. J. Farias, L. C. G. Freitas, "Retificador Híbrido Trifásico de Alta Potência e Elevado Fator de Potência com Reduzida DHTi Utilizando o Conversor Boost para Promover Suportabilidade a Afundamentos de Tensão", Eletrônica de Potência - SOBRAEP, Vol. 17, No. 3, pp. 609-622, Aug. 2012.

[21] G. B. Lima, D. B. Rodrigues, L. C. Freitas, E. A. A. Coelho, V. J. Farias, L. C. G. Freitas, "Novel Proposal of Hybrids Rectifiers with Voltage Sag Ride-Through Capability Based on Series DC Voltage Compensation Technique", in Proc. of IEEE Applied Power Electronics Conference and Exposition, pp 2162-2170, Mar. 2013.

[22] C. H. I. Font, I. Barbi, "Modelagem e Controle de um Retificador Trifásico Híbrido com Elevado Fator de Potência e Capacidade de Regeneração de Energia”, Eletrônica de Potência - SOBRAEP, Vol. 19, No. 1, pp. 47-57, Feb. 2014.

[23] A. A. Badin, G. D. Pinheiro, R. Gules, E. R. F. Romaneli, "Retificador Trifásico Abaixador Utilizando Dois Módulos Monofásicos Com Autotransformador", Eletrônica de Potência - SOBRAEP, Vol. 20, No. 1, pp. 85-93, Feb. 2015.

[24] K. Ogata, Engenharia de Controle Moderno, 3 ed., Rio de Janeiro: LTC, 2000.

\section{DADOS BIOGRÁFICOS}

Danillo Borges Rodrigues, nascido em Uberlândia (MG), em 1986, é engenheiro eletricista (2011), mestre (2013) e doutor (2016) pela Universidade Federal de Uberlândia (UFU FEELT). Atualmente é professor pela Universidade Federal do Triângulo Mineiro. Suas áreas de interesse incluem retificadores híbridos, técnicas de controle digitais, mitigação de conteúdos harmônicos de corrente, compensação de afundamentos de tensão e aplicações da eletrônica de potência em microrredes CC.

Paulo Ricardo da Silva, nascido em Araxá (MG), em 1991, é engenheiro eletricista (2015) pela Universidade Federal do Triângulo Mineiro (UFTM) e mestre (2018) pela Universidade Federal de Uberlândia (UFU - FEELT). Suas áreas de interesse incluem retificadores híbridos, técnicas de controle utilizando DSP e aplicações da eletrônica de potência em sistemas de geração distribuída baseados em fontes alternativas de energia. 
$\underline{\text { Luiz Carlos de Freitas, }}$ nascido em Monte Alegre (MG), em 1952, é engenheiro eletricista (1975) pela Universidade Federal de Uberlândia - Faculdade de Engenharia Elétrica (UFU-FEELT, Uberlândia / MG) mestre (1985) e doutor (1992) em Engenharia Elétrica pela Universidade Federal de Santa Catarina - Instituto de Eletrônica de Potência (UFSCINEP), Florianópolis (SC). Atualmente é professor titular da Faculdade de Engenharia Elétrica (FEELT) da UFU, Bolsista de Produtividade em Pesquisa $1 \mathrm{~B}$ do $\mathrm{CNPq}$, vinculado ao Núcleo de Eletrônica de Potência (NUPEP) da mesma Universidade. Possui 54 artigos publicados em periódicos nacionais e internacionais, e mais de 200 artigos publicados em conferências relacionados ao desenvolvimento de conversores CC-CC, CC-CA e CA-CC com comutação suave e retificadores multipulsos. Orientou 20 dissertações de mestrados e seis teses de doutorado como orientador principal. Desde 1996 é pesquisador com bolsa de produtividade em pesquisa do CNPq. O professor Luiz Carlos de Freitas publicou no PESC'92, no APEC'93, no PESC'93 e na IEEE Transactions on Power Electronics - janeiro/1995, a evolução de células de comutação suave que promovem a entrada e saída de condução de interruptores com tensão zero, as quais têm sido amplamente utilizadas em pesquisas relativas à Eletrônica de Potência desde então.

Gustavo Brito de Lima, nascido em Ibiassucê (BA), em 1986, é engenheiro eletricista (2009), mestre (2011) e doutor (2015) pela Universidade Federal de Uberlândia (UFUFEELT). Atualmente é professor da Universidade Federal de
Uberlândia. Suas áreas de interesse incluem retificadores híbridos e técnicas de controle analógicas e digitais.

Ernane Antônio Alves Coelho, nascido em Teófilo Otoni, Minas Gerais, em 1962, é engenheiro eletricista (1987) pela Universidade Federal de Minas Gerais, mestre (1989) pela Universidade Federal de Santa Catarina e doutor (2000) pela Universidade Federal de Minas Gerais. Atualmente é professor da Universidade Federal de Uberlândia. Sua área de atuação envolve inversores PWM, paralelismo de inversores, correção do fator de potência e novas tecnologias usando controle digital.

Luiz Carlos Gomes de Freitas, nascido em Uberlândia - MG em 1976, é engenheiro eletricista (2001), mestre (2003) e doutor (2006) pela Universidade Federal de Uberlândia Faculdade de Engenharia Elétrica (UFU - FEELT). Atualmente é professor associado da Faculdade de Engenharia Elétrica da UFU, vinculado ao Núcleo Pesquisa em Eletrônica de Potência (NUPEP), e bolsista de Produtividade em Pesquisa 2 do CNPq. Sua área de atuação é eletrônica de potência e suas áreas de interesse incluem qualidade da energia elétrica, correção ativa do fator de potência, técnicas de comutação não dissipativa, conversores CC-CC e CA-CC, inversores, retificadores híbridos, técnicas de controle utilizando DSP e aplicações da eletrônica de potência em sistemas de geração distribuída. Em 2012 recebeu o Prize Paper Award from IEEEIAS-Industrial Automation and Control Committee por sua contribuição no desenvolvimento de retificadores híbridos. 